\title{
Imported and Storm-Generated Near-Ground Vertical Vorticity in a Simulated Supercell*
}

\author{
JOHANNES M. L. DAHL ${ }^{+}$AND MATTHEW D. PARKER \\ Department of Marine, Earth, and Atmospheric Sciences, North Carolina State University, Raleigh, North Carolina
}

LOUIS J. WICKER

NOAA/National Severe Storms Laboratory, Norman, Oklahoma

(Manuscript received 29 April 2013, in final form 2 April 2014)

\begin{abstract}
The authors use a high-resolution supercell simulation to investigate the source of near-ground vertical vorticity by decomposing the vorticity vector into barotropic and nonbarotropic parts. This way, the roles of ambient and storm-generated vorticity can be isolated. A new Lagrangian technique is employed in which material fluid volume elements are tracked to analyze the rearrangement of ambient vortex-line segments. This contribution is interpreted as barotropic vorticity. The storm-generated vorticity is treated as the residual between the known total vorticity and the barotropic vorticity.

In the simulation the development of near-ground vertical vorticity is an outflow phenomenon. There are distinct "rivers" of cyclonic shear vorticity originating from the base of downdrafts that feed into the developing near-ground vortex. The origin of these rivers of vertical vorticity is primarily horizontal baroclinic production, which is maximized in the lowest few hundred meters AGL. Subsequently, this horizontal vorticity is tilted upward while the parcels are still descending. The barotropic vorticity remains mostly streamwise along the analyzed trajectories and does not acquire a large vertical component as the parcels reach the ground. Thus, the ambient vorticity that is imported into the storm contributes only a small fraction of the total near-ground vertical vorticity.
\end{abstract}

\section{Introduction}

It has long been known that mesocyclogenesis above the surface is achieved by the reorientation of preexisting horizontal vortex lines within the convective updraft (Rotunno and Klemp 1982; Davies-Jones 1984). The development of rotation at ground level, ${ }^{1}$ on the

\footnotetext{
${ }^{1}$ Technically, the velocity (and vertical vorticity) is zero at the ground $(z=0)$, so the terms "ground-level rotation" or "nearground rotation" are used to denote rotation at an arbitrarily small distance above the surface.
}

* Supplemental information related to this paper is available at the Journals Online website: http://dx.doi.org/10.1175/JAS-D-130123.s1.

${ }^{+}$Current affiliation: Department of Geosciences, Texas Tech University, Lubbock, Texas.

Corresponding author address: Dr. Johannes Dahl, Texas Tech University, Atmospheric Science Group, Box 41053, Lubbock, TX 79409.

E-mail: johannes.dahl@ttu.edu other hand, cannot be achieved by mere upward tilting of initially horizontal vortex lines, but a downdraft is required, as discussed by Davies-Jones (1982), DaviesJones and Brooks (1993), Walko (1993), Markowski and Richardson (2010), and Davies-Jones and Markowski (2013). The reason is that an initially horizontal nearground vortex line is lifted away from the surface as it is reoriented into the vertical at the updraft edge.

One way that a downdraft facilitates the development of vertical vorticity at the surface is by baroclinic production of horizontal vorticity at the downdraft periphery, followed by tilting and downward advection, as suggested by full-physics simulations (Rotunno and Klemp 1985; Davies-Jones and Brooks 1993) and observations (Markowski et al. 2008, 2012). However, in idealized, axisymmetric simulations, near-ground rotation is achieved "barotropically" (i.e., by reorienting preexisting vortex lines at the downdraft edge; DaviesJones et al. 2001; Markowski et al. 2003b; Davies-Jones 2008; Parker 2012). This barotropic vorticity corresponds to vorticity that is imported into the storm from the environment. In these simulations the baroclinic mechanism is, by symmetry, ineffective. Although analyses of 
3D full-physics simulations and observations suggest that the baroclinic mechanism is relevant, the barotropic contribution is not usually discussed explicitly (e.g., Rotunno and Klemp 1985; Wicker and Wilhelmson 1995; Adlermann et al. 1999). ${ }^{2}$

However, even in cases where the importance of baroclinity is evident, one might still ask whether the ambient, preexisting horizontal vorticity plays a direct role in near-ground rotation of supercells (irrespective of whether additional vorticity is produced baroclinically). One motivation for this question is that the ambient vertical wind shear at low levels is an important predictor for tornadoes (Markowski et al. 2003a). Moreover, whenever there are horizontal vortex lines that interact with a downdraft, one would expect the vortex lines to be depressed, potentially contributing to vertical vorticity at the surface. A quantification of the different sources of vertical vorticity in surface mesocyclones is crucial for understanding the genesis, maintenance, and decay of tornadoes.

The purpose of this study is to isolate the roles of the ambient and storm-generated vorticity in surface mesocyclogenesis within a simulated supercell. To achieve this goal, we decompose the vorticity into barotropic and "nonbarotropic" parts. ${ }^{3}$ The barotropic vorticity is formulated in Lagrangian coordinates, consistent with Cauchy's vorticity formula (Dutton 1976; Davies-Jones 2000; Davies-Jones 2006), which is implemented using a new technique whereby material volume elements and their geometric configuration are tracked and analyzed. Barotropic vorticity dynamics is much easier to interpret in the Lagrangian perspective than in the Eulerian picture. Using this approach, the rearrangement of existing vorticity and production of additional vorticity can be separated and quantified. The decomposition of vorticity into barotropic and nonbarotropic parts has been applied to orographic vortices by Epifanio and Durran (2002). Davies-Jones (2000) calculated baroclinic and barotropic vorticity in an analytical model that is applicable to dry downdrafts, but to our knowledge this approach has not been used in a full-physics representation of a supercell.

Section 2 summarizes the concepts of barotropic and nonbarotropic vorticity and how these ideas can be used to isolate the effects of ambient vorticity and

\footnotetext{
${ }^{2}$ Two exceptions are Davies-Jones and Brooks (1993), who discuss qualitatively the role of baroclinic and barotropic processes in full-physics simulations, and Markowski et al. (2012), who consider barotropic and baroclinic effects via the nonconservation of the circulation of a material circuit.

${ }^{3}$ Herein, nonbarotropic vorticity refers to the part of vorticity that is not due to the rearrangement of preexisting vortex lines.
}

storm-generated vorticity. Section 3 covers details about the simulation, and section 4 presents the results, which are further discussed in section 5. Conclusions are offered in section 6 .

\section{Separation of storm-generated and ambient vorticity}

From Helmholtz's second law (e.g., Truesdell 1954, p. 88), it follows that in inviscid, barotropic fluids, vortex lines are material lines. That is, a chain of fluid parcels making up a vortex line at one point in time is part of the same vortex line at any other time. An equivalent statement is that vortex lines are "frozen" into the fluid, behaving like elastic strings while being stretched and deformed. The local vorticity thus changes only as a result of advection, reorientation, and stretching of existing vortex lines. ${ }^{4}$ Per definition, vorticity vectors are tangent to vortex lines. In compressible fluids like air, a vortex-line segment behaves like the vorticity vector $\boldsymbol{\omega}$ divided by density $\rho, \mathbf{w}=\boldsymbol{\omega} / \rho$, which remains frozen in the fluid [see appendix A and also Davies-Jones (2006)]. That is, $\boldsymbol{\omega}$ itself is not in general frozen into the medium-its magnitude increases (decreases) as a result of $3 \mathrm{D}$ compression (expansion) relative to a perfectly frozen vector. This effect is appreciable in the present application as the parcels undergo substantial vertical excursions.

If the fluid is viscous or baroclinic, new vortex lines may be created (or existing ones destroyed), so that vortex lines are no longer material lines (e.g., Truesdell 1954, chapter 8). However, the vorticity field has a remarkable property (e.g., Dutton 1976, chapter 10; Davies-Jones 2000, 2006): even in viscous or baroclinic flows there is always one part of the vorticity that behaves as though the flow was inviscid and barotropic (existing vortex lines being rearranged). The other part is due to the production and subsequent rearrangement of additional vortex lines. The first part is commonly referred to as barotropic vorticity. The second part is due to the production of vorticity due to baroclinity and viscosity (and subsequent rearrangement) and will be referred to as nonbarotropic vorticity. The barotropic part is the vorticity that would result if vorticity generation by baroclinity or diffusion were "switched off" (Davies-Jones and Brooks 1993; Davies-Jones 2006). To apply this concept, an arbitrary initial "reference", time $t_{0}$ is chosen at which the entire vorticity is defined to be barotropic:

\footnotetext{
${ }^{4}$ These three contributions will be referred to as "rearrangement" of vortex lines.
} 


$$
\boldsymbol{\omega}_{\mathrm{BT}}\left(t_{0}\right)=\boldsymbol{\omega}\left(t_{0}\right),
$$

where $\boldsymbol{\omega}_{\mathrm{BT}}$ is the barotropic vorticity. The choice of $t_{0}$, then, determines what is meant by the barotropic part of the vorticity. The nonbarotropic vorticity $\boldsymbol{\omega}_{\mathrm{NBT}}$ is defined to be zero at $t_{0}$ :

$$
\boldsymbol{\omega}_{\mathrm{NBT}}\left(t_{0}\right)=\mathbf{0} .
$$

At time $t$, the total vorticity $\boldsymbol{\omega}(t)$ is given by the sum of rearranged barotropic vortex lines and the vortex lines subsequently generated by baroclinic/diffusive effects (and then rearranged):

$$
\boldsymbol{\omega}(t)=\boldsymbol{\omega}_{\mathrm{BT}}(t)+\boldsymbol{\omega}_{\mathrm{NBT}}(t) .
$$

Here and in the following all variables represent Lagrangian quantities following the fluid motion. For instance, the symbol $\boldsymbol{\omega}(t)$ is a short-hand notation for $\boldsymbol{\omega}_{\mathbf{a}}(t)$ and represents the vorticity of a parcel with the label $\mathbf{a}$.

\section{a. Mathematical formulation}

The barotropic vorticity of a parcel is given by Cauchy's vorticity formula, which may be written symbolically as (e.g., Casey and Naghdi 1991)

$$
\boldsymbol{\omega}_{\mathrm{BT}}(t)=\Pi\left\{\boldsymbol{\omega}\left(t_{0}\right)\right\} .
$$

The "input" for this equation is the vorticity at the reference time $\boldsymbol{\omega}\left(t_{0}\right)$. The action of the linear transformation symbolized by the $\Pi$ operator sends the vorticity vector forward in time, yielding the barotropic vorticity at time $t$. This is an "active" interpretation of the transformation: The vector at $t_{0}$ itself is transformed to yield the vector at time $t$. This is perhaps the most common interpretation of this formula (e.g., Casey and Naghdi 1991; Epifanio and Durran 2002; Davies-Jones 2006).

Equivalently, we may interpret Cauchy's formula as a "passive" transformation, whereby the basis vectors are changed while the barotropic vorticity vector itself remains the same. This perspective is entirely consistent with the customary active viewpoint, but we introduce it because it clearly reveals how the barotropic vorticity vector behaves within a material fluid element. Following Aris (1962, p. 77) and Salmon (1998, p. 5 and p. 201), we define the fixed space (represented by the model grid) as "Eulerian space," which is endowed with the fixed Cartesian coordinates referred to as Eulerian (or spatial) coordinates. We also define a coordinate system that is moving with the flow, known as the Lagrangian (or material) coordinate system $a^{\mu}(\mu=1,2,3)$. This system is "dragged" through the fixed Eulerian space. As the Lagrangian coordinate grid materially follows the flow, it becomes deformed, behaving like a deformable continuum itself. The material coordinate system is defined by the locations of the fluid parcels at $t_{0}$, which serve as labels that remain attached to each parcel. That is, at $t_{0}$ the Lagrangian and Eulerian frames coincide. As discussed in the next section, in this study the Lagrangian frame is set up by fluid parcels launched within the simulation. Since the Lagrangian frame becomes deformed in time, there are two sets of coordinate basis vectors. The covariant basis is given by $\mathbf{g}_{\mu}=\partial \mathbf{r} / \partial a^{\mu}$, where $\mathbf{r}$ is the position vector. The covariant basis vectors are parallel to the local coordinate lines, and they are deformed in the same manner as the fluid volume itself. This basis is time dependent and naturally reflects the deformation of the fluid volume. Greek indices (always running from 1 to 3 ) always pertain to this covariant basis. The corresponding vector components are contravariant and denoted by superscripts, so that $\mathbf{w}=$ $\mathbf{g}_{\mu} w^{\mu}$, where Einstein's summation convention is used. On the other hand, the contravariant basis is given by $\mathbf{g}^{\nu}$ and is orthogonal to the local coordinate lines $a^{\nu}$. We will only use the covariant basis because it intuitively reflects the deformation of the coordinate frame (Figs. 1a,b). In the following, "Lagrangian basis" always refers to the time-dependent, covariant basis of the curvilinear Lagrangian coordinate system. The reader is referred to Simmonds (1994) for more background on curvilinear coordinate systems.

The other basis we will use is the standard Cartesian basis, given by $\left\{\mathbf{e}^{i}\right\}=\left\{\mathbf{e}_{i}\right\}$. The Cartesian coordinates are given by $r^{i}\left(=r_{i}\right)$ and are signified by Latin indices (running from 1 to 3 ). As we follow a given parcel labeled $\mathbf{a}$ and observe the evolution of its vorticity vector, we may always switch from the covariant basis belonging to the curvilinear Lagrangian coordinates to the Cartesian basis (the Lagrangian perspective does not prescribe the basis vectors relative to which vector components are expressed). The advantage of introducing the Lagrangian basis is that a vector such as $\mathbf{w}$ that is frozen into the medium always has the same components, no matter how strongly the fluid volume is deformed. The reason is that the deformation is contained in the timedependent covariant basis. ${ }^{5}$ The barotropic vorticity vector itself is not frozen into the medium, but its contravariant components still take a rather simple form when expressed with respect to the Lagrangian basis. These components may be inferred from Cauchy's formula, which may be interpreted simply as transformation from the Lagrangian basis to the Cartesian basis (see appendix A):

\footnotetext{
${ }^{5}$ This is why we chose the covariant basis, rather than the contravariant basis, on which the $\mathbf{w}$ components are not conserved.
} 
a) Reference configuration at $t_{0}$ (Lagrangian $=$ Cartesian frame)

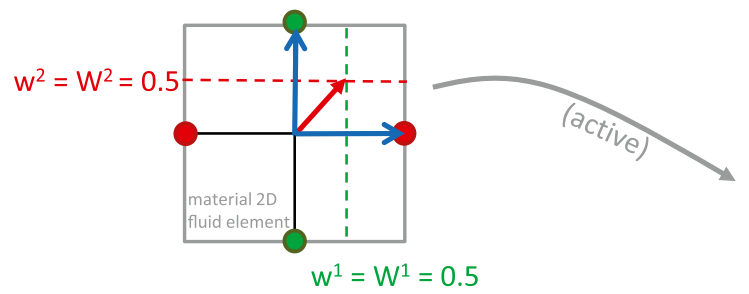

c) Configuration at $\mathrm{t}$ (Cartesian frame)

b) Configuration at $\mathrm{t}$ (deformed Lagrangian frame)
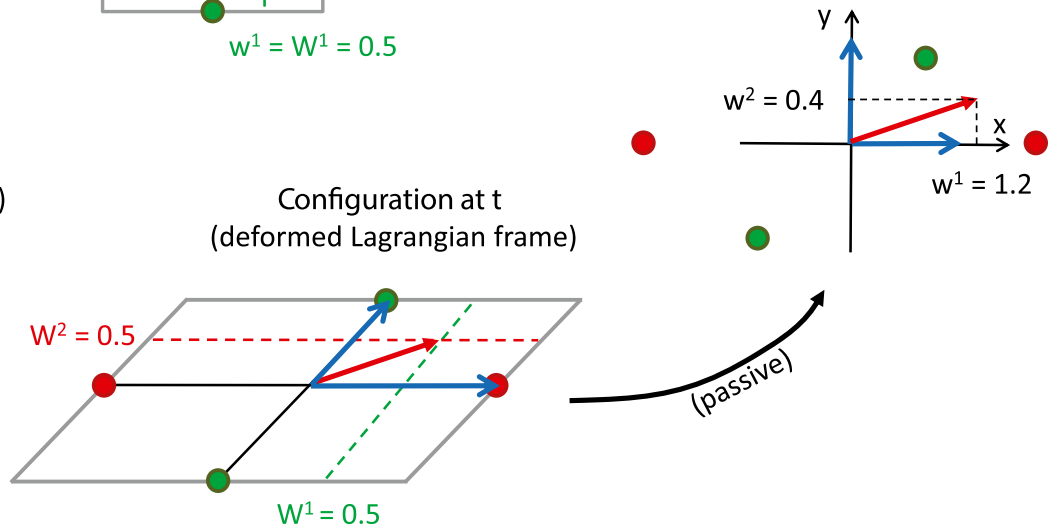

FIG. 1. Two-dimensional depiction of the relation between the reference configuration and the configuration at time $t$, in time-dependent Lagrangian coordinates and Cartesian coordinates. The blue arrows represent the covariant basis vectors. The green and red circles are the stencil parcels and the red arrow represents a vector that is frozen into the fluid element. (a) At the reference time, this vector is given by its components, $\left[w^{1}, w^{2}\right]=\left(W^{1}, W^{2}\right)=(0.5,0.5)$ and the Lagrangian frame coincides with the Cartesian frame. Here, $w^{i}$ represent the components with respect to the fixed Cartesian basis and $W^{\alpha}$ represent the components with respect to the time-dependent covariant Lagrangian basis. (b) Although the fluid element is deformed, the Lagrangian coordinates $\left(W^{1}, W^{2}\right)$ of the vector remain constant (the blue basis vectors become deformed as the Lagrangian frame is dragged through Eulerian space). (c) A coordinate transformation [curved black arrow connecting (b) and (c)] yields the desired components in Cartesian coordinates, $\left(w^{1}, w^{2}\right)=(1.2,0.4)$. The vector itself remains unchanged during this transformation.

$$
\omega_{\mathrm{BT}}^{i}(t)=\frac{\partial r^{i}}{\partial a^{\nu}} \Omega_{\mathrm{BT}}^{\nu}(t)
$$

where $\omega_{\mathrm{BT}}^{i}$ represents the parcel's vorticity components on the Cartesian basis, $\Omega_{\mathrm{BT}}^{\nu}$ represents the parcel's vorticity components on the covariant Lagrangian basis at time $t$, and Einstein's summation convention is in force. It then follows (appendix A) that the barotropic vorticity components $\Omega_{\mathrm{BT}}^{\nu}$ in the Lagrangian frame are given by

$$
\Omega_{\mathrm{BT}}^{\nu}(t)=\left[J_{\mathbf{a}}^{\mathbf{r}}(t)\right]^{-1} \omega_{0}^{\nu},
$$

where $J_{\mathbf{a}}^{\mathbf{r}}$ is the Jacobian of the transformation matrix from the Lagrangian basis to the Cartesian basis and $\omega_{0}^{\nu} \equiv\left[\boldsymbol{\omega} \cdot \mathbf{g}^{\nu}\right]_{t_{0}}=\boldsymbol{\omega}\left(t_{0}\right) \cdot \mathbf{e}^{\nu}$ is the vorticity component in the $a^{\nu}=r^{\nu}$ direction at the reference time. Because we choose the initial Lagrangian frame to be Cartesian, $\Omega_{\mathrm{BT}}^{\nu}$ simply represents the Cartesian components of the curl of the velocity field at $t_{0}$, multiplied by $\left[J_{\mathbf{a}}^{\mathbf{r}}(t)\right]^{-1}=\rho(t) / \rho\left(t_{0}\right){ }^{6}$ At $t_{0}$, the barotropic vorticity is just the curl of the velocity field, because then $J_{\mathbf{a}}^{\mathbf{r}}=1$, consistent with Eq. (1). This interpretation immediately reveals the behavior of barotropic vorticity following a parcel: if the flow is incompressible, $J_{\mathbf{a}}^{\mathbf{r}}=1$ and $\Omega_{\mathrm{BT}}^{\nu}=$ const. The barotropic vorticity is thus frozen into the medium. In this case, the components of the vector are conserved with respect to the time-dependent covariant Lagrangian basis, no matter how strongly the coordinate grid is deformed. The reason is that the vector is stretched and reoriented in the same fashion as the local coordinate frame. This is

\footnotetext{
${ }^{6}$ The relation between the Jacobian and the density ratio may be inferred from transforming a material volume element $d^{3} \mathbf{a}$ to Eulerian coordinates: $d^{3} \mathbf{r}=J_{\mathbf{a}}^{\mathbf{r}} d^{3} \mathbf{a}$. Since mass is conserved, it immediately follows that $\rho / \rho_{0}=\left[J_{\mathbf{a}}^{\mathbf{r}}\right]^{-1}$. See Aris $(1962$, p. 83) for more discussion.
} 
demonstrated for a 2D example of a material line segment in Figs. 1a and 1b. To obtain the desired Cartesian components of the vector tangent to that line segment, the transformation matrix is applied (Fig. 1c). This transformation leaves the vector unchanged; merely its components are expressed with respect to the Cartesian basis (rather than the covariant Lagrangian basis). In compressible flows, the barotropic vorticity vector is no longer perfectly frozen into the fluid, so the Lagrangian components $\Omega_{\mathrm{BT}}^{\nu}$ are no longer conserved, which is reflected by the time-dependent Jacobian.

Conceptually, the advantage of this approach is that one is forced to think of barotropic vorticity as a $3 \mathrm{D}$ vector that is reoriented and stretched within the deformable medium. Traditionally, the vorticity evolution is analyzed using the vorticity equation and evaluating each term in the equation separately for each Cartesian component. This may sometimes complicate the conceptualization of the involved processes, especially because the "stretching" terms may also reorient vortex lines and "tilting" terms also stretch the vortex lines, as demonstrated in Fig. 2 (see also Davies-Jones 1982).

The foregoing discussion highlights barotropic vorticity dynamics as a purely geometric problem. One merely needs to track the local geometric configuration of the fluid and determine its initial vorticity. One is then able to calculate the barotropic vorticity. Next we describe how these concepts can be applied to rotation in supercells.

\section{b. Application}

\section{1) BAROTROPIC VORTICITY}

Assume that at some point in time, the vorticity of a parcel that is still in the far field of the storm and that eventually becomes part of the near-ground vorticity extremum is known. Let this parcel be surrounded by six neighbors, all initially aligned along the three Cartesian axes. These six parcels define a 3D "stencil" around the parcel whose barotropic vorticity is to be determined. With time, the coordinate stencil, which represents the Lagrangian coordinates, is deformed and stretched or compressed, and the embedded barotropic vorticity vector is reoriented and stretched accordingly. These stencil parcels are represented by the red and green circles in Fig. 1. (In the figure, the vertical dimension of the stencil is suppressed for clarity).

To obtain the components of barotropic vorticity in Cartesian coordinates, only the initial vorticity needs to be known, as well as the transformation matrix from the curvilinear to the Cartesian coordinates (and its Jacobian) at the time of interest. This matrix is obtained from the locations of the stencil parcels, and it is approximated by a) $\begin{aligned} & \mathrm{T}>0 \\ & \mathrm{~S}\end{aligned}=0$

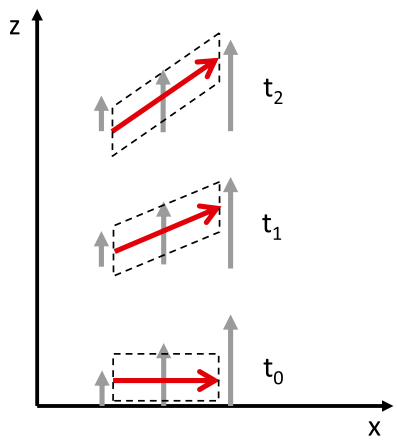

b) $\quad T=0$

$S>0$

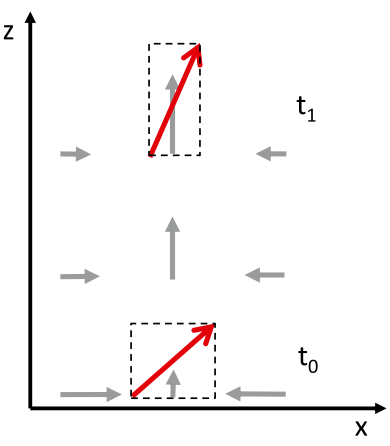

FIG. 2. Demonstration that (a) the tilting term also elongates the barotropic vortex-line segment and (b) the stretching term may also reorient the barotropic vortex-line segment (here shown for an incompressible flow). The vortex-line segment is shown in red as it rises through the depicted flow regimes for successive times. The arrowhead is pointing in the direction of the vorticity. The background velocity is constant in time and represented by gray arrows. The dashed black outlines represent the material volume in which the vortex line is embedded and that is deformed in the flow. The tilting term is $T=\boldsymbol{\omega}_{h} \cdot \nabla_{h} w$ and the stretching term is $S=-\zeta\left(\nabla_{h} \cdot \mathbf{v}_{h}\right)$, where $\boldsymbol{\omega}_{h}$ is the horizontal vorticity, $\nabla_{h}$ is the horizontal gradient operator, and $\mathbf{v}_{h}$ is the horizontal velocity vector.

$$
\frac{\partial r^{k}}{\partial a^{\nu}} \approx \frac{\Delta r^{k}\left(a^{\nu}\right)}{\Delta a^{\nu}},
$$

where $\Delta r^{k}\left(a^{\nu}\right)$ is the time-dependent distance along the $r^{k}$ axis between the two stencil parcels that were initially aligned along the $r^{\nu}\left(=a^{\nu}\right.$ at $\left.t_{0}\right)$ axis. The quantity $\Delta a^{\nu}$ is the distance between these parcels along the $a^{\nu}$-coordinate axis at reference time, which is constant with $\Delta a^{\nu}=2 \mathrm{~m}$. In this treatment, it is implied that the vortex-line segment in the coordinate stencil as well as the coordinate lines are straight. Also, the coordinate lines are assumed to cross the central parcel of the stencil. As will be shown in section 4, the relative distances between the parcels are only a few meters through the analysis period, so that this approximation is justified.

Computationally, an advantage of the new technique is that the barotropic vorticity only depends on the initial vorticity and the geometric configuration of the fluid (reflected by the transformation matrix and its Jacobian). In contrast, direct numerical integration of the barotropic forcing of the vorticity equation along trajectories easily leads to small interpolation errors in the forcing functions-involving products of spatial derivatives, which in addition are prone to discretization errors-which tend to accumulate with time. Also, errors may be incurred by inaccurate trajectories. Although trajectory errors may also affect the new technique, here the only remaining source of error is the finite-difference 
approximation, Eq. (7), which stays accurate unless the stencil becomes asymmetrically deformed. Regarding the implementation, apart from obtaining the location of the stencil parcels, only a few and rather simple calculations are required compared to the numerical integration of the barotropic forcing in the vorticity equation.

To identify the barotropic vorticity with the environmental vorticity in which the storm is embedded, the reference time must be selected such that the parcels are unperturbed by the storm. Otherwise, at least some stormgenerated vorticity would inadvertently be included in the barotropic vorticity (i.e., the vortex lines that are materially following the fluid may have been generated baroclinically within the storm), and we would no longer be able to separate ambient from storm-generated vorticity.

\section{2) NONBAROTROPIC VORTICITY}

One way of obtaining the nonbarotropic vorticity is via integrals of the vorticity equation (e.g., Dutton 1976; Mobbs 1981; Davies-Jones 2000; Epifanio and Durran 2002; Davies-Jones 2006). However, the vorticity at time $t$ is simply the sum of barotropic and nonbarotropic parts [Eq. (3)]. Since the total vorticity along the trajectories is known from model output, and the barotropic vorticity is calculated using Cauchy's formula, the nonbarotropic effects (baroclinity and diffusion) may be treated as the residual.

In summary, we calculate the barotropic vorticity along trajectories in Lagrangian coordinates and obtain its components in Cartesian coordinates using Cauchy's formula. This procedure is applied by tracking stencils of parcels and calculating changes in their geometric configuration using a finite-difference approximation. The barotropic vorticity is interpreted as ambient vorticity that is imported into (and rearranged within) the storm. The storm-generated (nonbarotropic) vorticity is simply the difference between the total and the barotropic vorticity.

\section{Methods}

\section{a. Model configuration}

We used an experimental version of the Bryan Cloud Model, version 1 (CM1; Bryan and Fritsch 2002), provided to the authors by G. Bryan. This version allows for highly accurate forward-trajectory calculations, as discussed below. The model was initialized with the Del City homogeneous base-state environment (Brandes 1981) and run forward for $5400 \mathrm{~s}^{7}$ The storm was

\footnotetext{
${ }^{7}$ In the interest of reproducibility, the base-state sounding is provided as online supplement.
}

initiated with an ellipsoidal $2.0-\mathrm{K}$ temperature perturbation. The equations are discretized on an Arakawa $\mathrm{C}$ grid with a horizontal grid spacing of $250 \mathrm{~m}$ and a vertical grid spacing varying from $100 \mathrm{~m}$ near the ground to $500 \mathrm{~m}$ toward the domain top, which is at $20 \mathrm{~km}$ AGL. The lowest scalar grid level [which coincides with the lowest level of the $(u, v)$ grid] is at $50 \mathrm{~m}$ AGL. The horizontal dimensions are $120 \mathrm{~km}$ in the $x$ and $y$ directions. The lateral boundary conditions are open, and free-slip conditions are employed at the top and bottom of the domain. A sponge layer is used in the upper $6 \mathrm{~km}$ to reduce the impact of gravity waves reflected downward at the top boundary. A constant storm-motion vector was subtracted from the base-state winds to keep the storm stationary. Thus, in the following all velocities pertain to the storm-relative frame.

A six-category single-moment bulk microphysics scheme (Gilmore et al. 2004) is used, where we reduced the rain intercept parameter to $1 \times 10^{8} \mathrm{~m}^{-4}$ to prevent overly large temperature deficits in the cold pool (Dawson et al. 2010). The subgrid-scale turbulence is parameterized using a 1.5 -order closure. The horizontal advection scheme is of sixth order, and a small amount of sixth-order artificial diffusion is added to control small-scale noise. No radiation or surface fluxes are included, and the Coriolis parameter is set to zero.

The simulation produces a supercell storm with typical features such as a hook echo, downdrafts north and west of the updraft, and regions of strong near-ground rotation (Fig. 3). By $5220 \mathrm{~s}$, an intense vortex with vertical vorticity $\zeta$ exceeding $0.1 \mathrm{~s}^{-1}$ (Fig. 4) has developed at the lowest model level. This vortex is fed by "rivers" of vertical vorticity within the outflow that emanate from downdrafts north and west of the incipient vortex (Fig. 5). Notably, there is an absence of appreciable vertical vorticity east of the cold pool. Our goal was to identify the sources of vertical vorticity within the $\zeta$ streamers that originate from downdrafts. The model was restarted at $4200 \mathrm{~s}, 17 \mathrm{~min}$ prior to the peak intensity of the vortex. In the restart run, 3484860 forward trajectories were launched upstream of the main downdraft (the initial parcel density was about $32000 \mathrm{~km}^{-3}$ ). We focused on the region upstream of the main downdraft based on numerous simulations and restart runs carried out over the course of this study that unambiguously identified this region as the source of air for the near-ground vertical vorticity maxima (see also Dahl et al. 2012). The parcels were integrated forward on every model time step $(2.0 \mathrm{~s})$ based on the resolved velocity field using a fourth-order RungeKutta scheme and a fourth-order Lagrange polynomial spatial interpolator. 


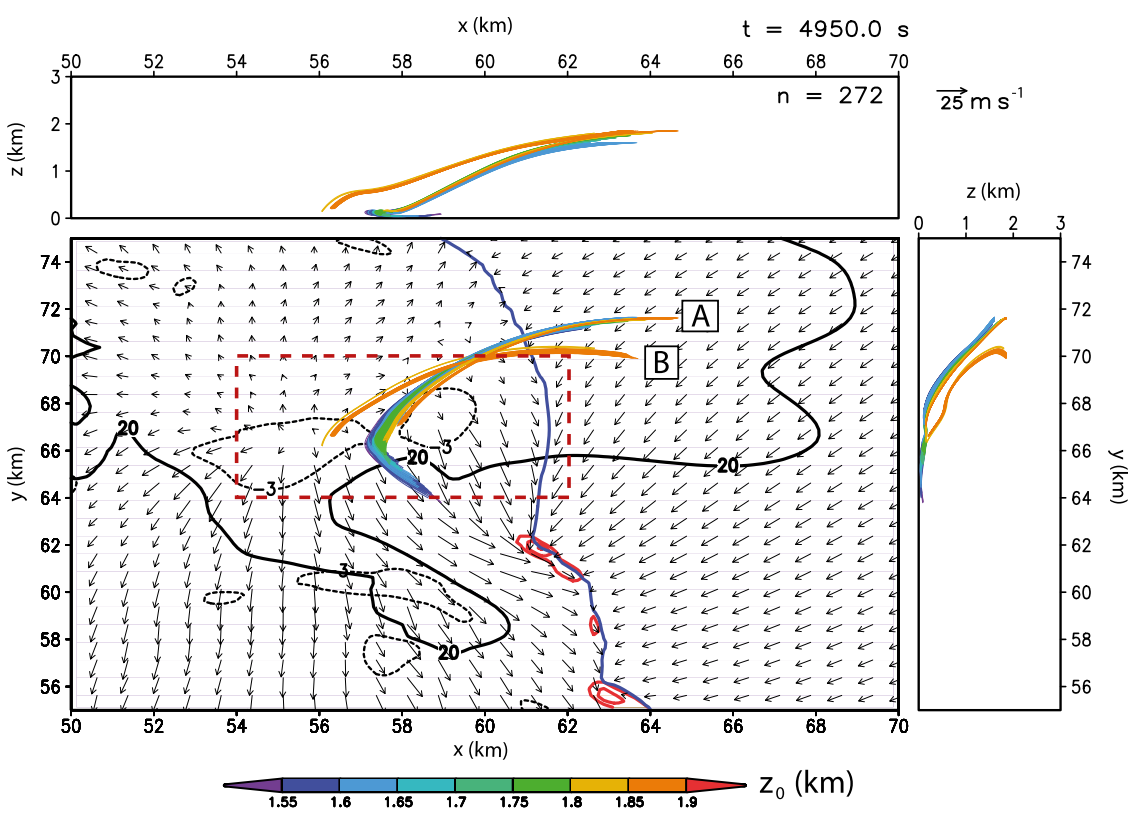

FIG. 3. The simulated supercell at $4950 \mathrm{~s}$. The solid black line is the $20-\mathrm{dB} Z$ reflectivity contour at $3375 \mathrm{~m}$ AGL. The dashed lines are the $-3 \mathrm{~m} \mathrm{~s}^{-1}$ vertical velocity contours at $375 \mathrm{~m}$ AGL, and the arrows are horizontal wind vectors at the lowest scalar grid level (50 $\mathrm{m}$ AGL). The blue line depicts the $-1-\mathrm{K}$ potential temperature perturbation, marking the gust-front position. Also plotted are the trajectories between 4200 and 4950 s that acquire positive vertical vorticity during descent (the color represents their initial altitude according to the color bar). The two sets of trajectories are labeled A and B, respectively. The red contours represent the 0.02, 0.04, 0.06, and $0.08 \mathrm{~s}^{-1}$ vertical vorticity isopleths at $50 \mathrm{~m}$ AGL. The red dashed square shows the spatial window in which parcels fulfilling the criteria described in the text were selected.

\section{b. Trajectories of interest}

Identifying trajectories that become part of the nearground vortex is rather challenging. Ideally, one would identify a threshold vertical vorticity value characteristic of the near-ground vortex and identify those parcels that reach this value within the space and time window of interest. However, as the parcels descend below the lowest scalar model level, a somewhat arbitrary choice must be made with respect to their horizontal velocities. This freedom implies that the parcels' horizontal velocity magnitude and direction are poorly constrained near the ground, as explained in the following. In the model, the zero-gradient lower boundary condition, $\partial u / \partial z=\partial v / \partial z=w=0$ at $z=0$, merely requires that the first scalar gridpoint above and beneath the ground are assigned the same value for each horizontal velocity component. However, there is no obvious choice for the vertical $(u, v)$ profile between these two scalar grid levels. Traditionally in free-slip simulations, the horizontal velocity is treated as constant beneath the physical scalar domain. However, this treatment is dynamically inconsistent for parcels, which thereby arbitrarily lose the part of the horizontal vorticity related to the vertical shear of the horizontal wind once they descend below the lowest scalar grid level (where the horizontal wind has been assumed constant with height). Alternatively, one might extrapolate the horizontal velocities downward to circumvent the partial loss of horizontal vorticity as the parcels approach the ground. This approach is still consistent with the implementation of the zero-gradient condition, and it is used in the current model implementation. It avoids the unrealistic horizontal vorticity evolution along trajectories as mentioned above. Also, it more faithfully preserves the cell-averaged fluxes (G. Bryan 2012, personal communication). However, this treatment is not optimal, either, as an inconsistency may arise between the velocities of the near-ground parcels and the advective motion of the near-ground vorticity field. For example, if a surface vertical vorticity maximum moved solely because of advection, the parcels' velocities at the surface relative to that maximum should be zero. The downward extrapolation of velocities, however, is in conflict with this behavior because at every time step the footprint of the vorticity field at the surface is determined by extrapolating the horizontal velocities at the lowest $(u, v)$ model levels downward. The motion of the surface vertical vorticity field is thus determined 


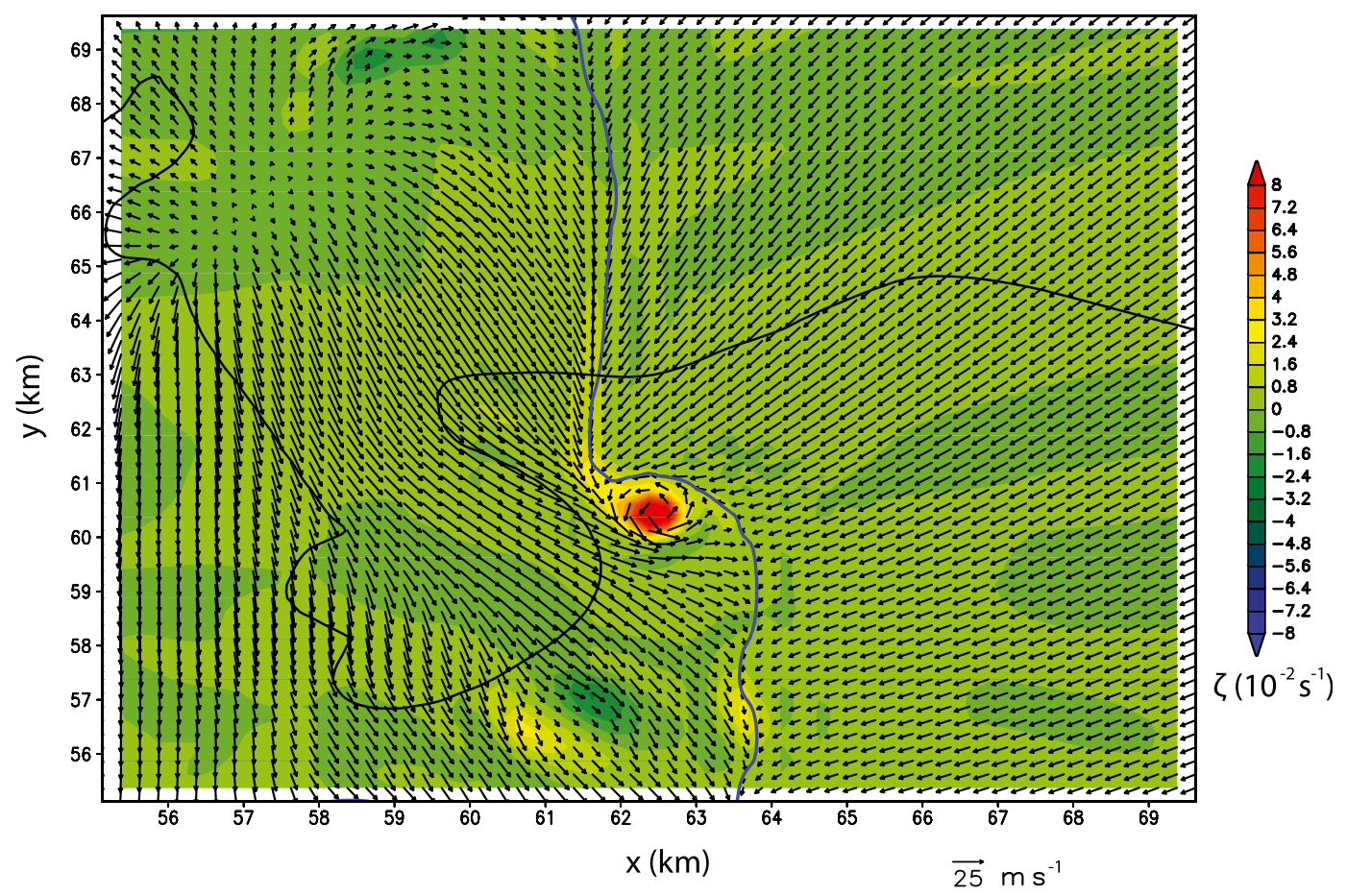

FIG. 4. Vertical vorticity field (shaded) and horizontal velocity vectors at $50 \mathrm{~m}$ AGL at $5220 \mathrm{~s}$ when the vortex reaches peak intensity $\left(\zeta>0.1 \mathrm{~s}^{-1}\right)$. To facilitate orientation, the 20 -dBZ reflectivity contour at $3375 \mathrm{~m}$ AGL (black line) and the $-1-\mathrm{K}$ potential temperature perturbation (blue line) are also shown.

by the flow at lowest model levels rather than by advection due to the extrapolated near-surface flow. This may potentially lead to unphysical vertical vorticity tendencies along trajectories (see appendix B). Also, the direction in which a parcel departs the downdraft base will be affected by the choice of the near-ground wind profile. This implies that whether or not a parcel reaches a vortex may directly depend on the treatment of the horizontal velocities beneath the scalar domain, with obvious effects on the parcel's vorticity evolution. To our knowledge, there currently is no agreed-upon physically consistent way of treating the lower boundary condition for parcels in free-slip simulations. We are thus skeptical of the near-ground behavior of the trajectories and chose to consider trajectories only within the scalar model domain (at or above the lowest scalar model level). Because we therefore cannot track parcels all the way into the surface vortex (more details are given in appendix B), we use physical constraints to identify the parcels of interest. The selection of parcels is based on the observation that at the lowest scalar model level, the vortex is fed by rivers and "lobes" of vertical vorticity that emanate from downdrafts north and west of the incipient vortex (Fig. 5). As the vertical vorticity is zero upstream of the storm, this suggests that parcels acquire vertical vorticity while descending within the downdraft.
This is consistent with previous findings that mere upward tilting of near-ground horizontal vortex lines interacting with updrafts cannot facilitate the development of an end-wall vortex (e.g., Davies-Jones 1982; DaviesJones and Brooks 1993; Walko 1993; Davies-Jones and Markowski 2013). We thus require the parcels to have achieved positive vertical vorticity as they pass through the lowest scalar model level (specifically, $\zeta>0.002 \mathrm{~s}^{-1}$ for $50 \leq z \leq 70 \mathrm{~m}){ }^{8}$ To focus on parcels close to the incipient vortex, we also defined a space-time window $(54 \leq x \leq 62 \mathrm{~km}, 64 \leq y \leq 70 \mathrm{~km}, 4500 \leq t \leq 5100 \mathrm{~s}$; red square in Fig. 3) and required that parcels move southward toward the developing vorticity maximum. This way, we captured about 4000 parcels that fulfilled the above criteria.

To ensure that the parcels' initial vorticity corresponds to the base-state vorticity, one would need to launch the trajectories far upstream of the storm, because the storm alters its environment with concomitant changes in the vorticity field. These modifications of the

\footnotetext{
${ }^{8}$ These values were chosen to limit the number of identified parcels; a larger $z$ interval or a smaller $\zeta$ threshold would have yielded tens of thousands of parcels. We adjusted the values such that a few thousand parcels resulted.
} 

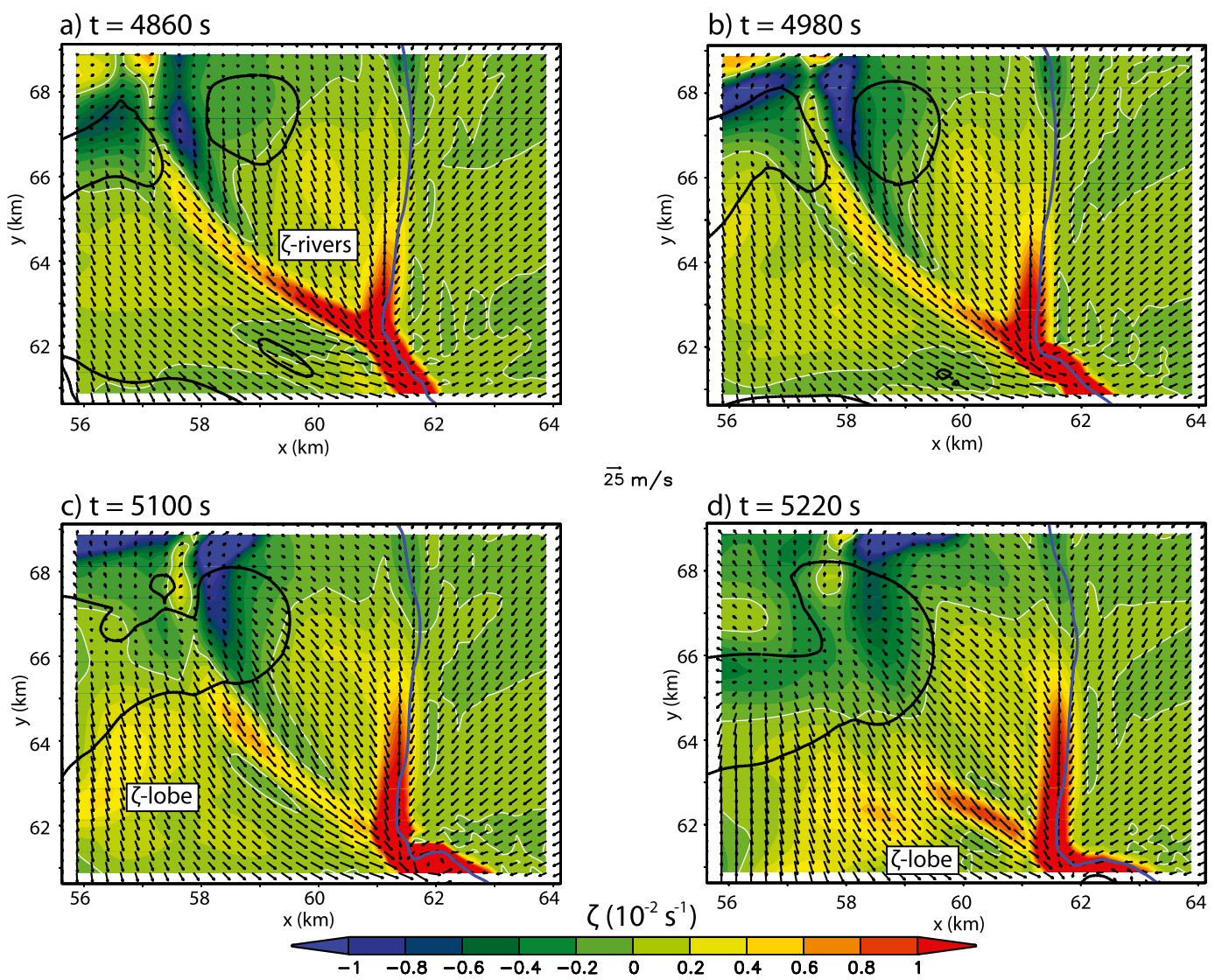

$\overrightarrow{25} \mathrm{~m} / \mathrm{s}$

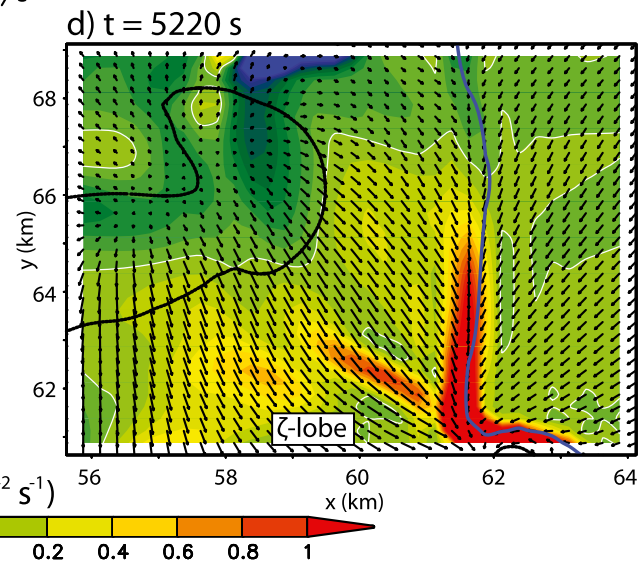

FIG. 5. Vertical vorticity (shaded) and horizontal velocity vectors at $50 \mathrm{~m}$ AGL at (a) 4860, (b) 4980, (c) 5100, and (d) $5220 \mathrm{~s}$. The white contour represents $\zeta=0$. A smaller interval of $\zeta$ is plotted compared to Fig. $4(-0.01 \leq \zeta \leq$ $0.01 \mathrm{~s}^{-1}$ ) to reveal the rivers of $\zeta$ streaming into the developing vortex. The black lines represent the $-2 \mathrm{~m} \mathrm{~s}^{-1}$ vertical velocity contour at about $266 \mathrm{~m}$ AGL and the blue line marks the cold-pool edge ( $-1-\mathrm{K}$ potential temperature perturbation).

storm's environment are related to the forward-flank baroclinic zone, gravity wave adjustment, and the effect of the perturbation-pressure field surrounding the storm's updraft. Although initializing the trajectories at $4200 \mathrm{~s}$ provides a comparatively long history of the parcels prior to reaching the ground, these parcels are still not far enough upstream of the storm to be completely unaffected by these perturbations. Launching the trajectories upstream of the midlevel forward-flank baroclinic zone would require some $50 \mathrm{~min}$ of forward integration, which is not feasible computationally given the large number of trajectories (and the number of restart runs that would be required to identify the initial locations of the parcels). Instead, we tested several ways of defining what constitutes "ambient vorticity" (e.g., base-state values or local averages in the vicinity of the storm). The results presented in the next section are based on those parcels whose initial horizontal-vorticity magnitude was perturbed by less than $10 \%$ from the base-state value and whose vertical vorticity was less than $0.001 \mathrm{~s}^{-1}$, yielding altogether 272 parcels. Although the number of identified parcels within each of the two bundles of trajectories (Fig. 3) is sensitive to these choices, the overall trajectory topology and behavior are robust. Reassuringly, this procedure of identifying suitable parcels yields trajectories that become part of the vorticity lobes and streamers emanating from the downdrafts (Fig. 6).

Although the selected parcels gain positive $\zeta$ close to the ground, they ascend in the updraft prior to reaching the near-ground vortex. As discussed in appendix B, we speculate this behavior to be related to the treatment of the upward velocity beneath the lowest model level. Also, in appendix B we discuss other criteria for selecting the parcels and why we have less confidence in analyzing those compared to the ones shown in Fig. 6 and discussed in the following. Once parcels of interest were identified, the simulation was restarted again at $4200 \mathrm{~s}$, this time including a stencil of six parcels surrounding each of the 272 parcels identified previously. 


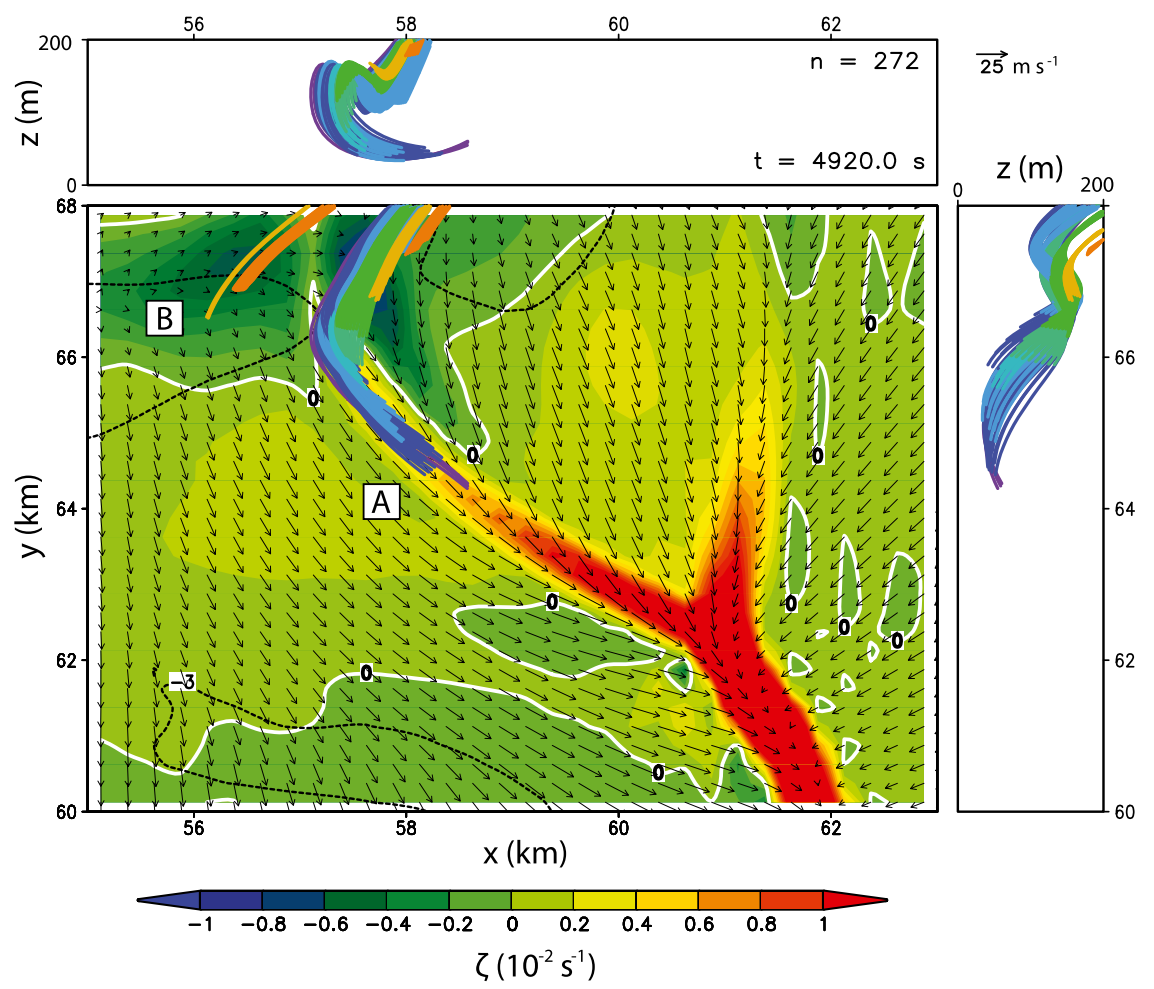

FIG. 6. As in Fig. 5, but at 4920 s. In addition, trajectories are shown, color coded as in Fig. 3. There are two sets of trajectories contributing to positive vertical vorticity, labeled A and B. At the time shown, trajectories within set B have not yet descended below $200 \mathrm{~m}$ and are thus not shown in the vertical projections. These trajectories eventually contribute to a lobe of positive vorticity west of the $\zeta$ river (Fig. 5).

These stencil parcels represent the local Lagrangian coordinates and are initially aligned along the Cartesian axes at a distance of $1 \mathrm{~m}$ from the central parcel (whose barotropic vorticity is to be determined). The restart time corresponds to the "reference time" used to define the initial barotropic and nonbarotropic vorticity [Eqs. (1) and (2)]. These parcels are used to track the Lagrangian coordinate system and to calculate the transformation from the covariant Lagrangian basis to the Cartesian bases, as described above.

\section{Results}

There are two sets of trajectories that acquire positive vertical vorticity during descent, labeled A and B in Figs. 3 and 6. Fundamentally, these two sets are not different from each other-parcels in set B merely do not undergo as much vertical stretching following their descent as parcels in set $\mathrm{A}$, thus resulting in a comparatively weak lobe of positive $\zeta$ rather than a compact $\zeta$ extremum (Figs. 5 and 6). In the following we show the vorticity evolution of a representative trajectory within sets A and B. To support the generality of the results, we also show averages and vorticity distributions over all 272 parcels.
Figure 7 shows the total vorticity vector along a representative trajectory within set A over the course of $12 \mathrm{~min}$. The parcel acquires appreciable negative vertical vorticity during its initial descent. As it approaches the ground, however, it attains a weak upward (cyclonic) vorticity component (Fig. $7 b$ ), which gives rise to the near-ground $\zeta$ river whose origin we seek to understand. To this end, Fig. 8 depicts the storm-generated part of the vorticity. Initially zero, it acquires a significant antistreamwise component as the parcel descends through the northern periphery of a buoyancy minimum in the storm's forward flank (Fig. 9a). However, as the parcel reaches a downdraft core farther downstream at an altitude of approximately $300 \mathrm{~m} \mathrm{AGL}$, it acquires large crosswise storm-generated vorticity that becomes increasingly streamwise as the parcel is pushed eastward by the pressure gradient force associated with the downdraft base. ${ }^{9}$ This conversion from crosswise to streamwise vorticity is a combination of two effects. First, the parcel

\footnotetext{
${ }^{9}$ Since the storm-generated (nonbarotropic) vorticity is due mainly to baroclinic production (rather than diffusion), we will refer to it as baroclinic vorticity.
} 
a)

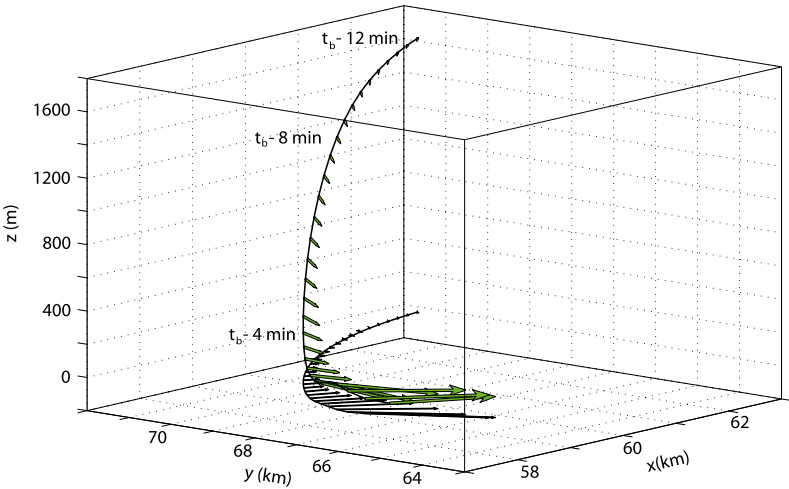

b)

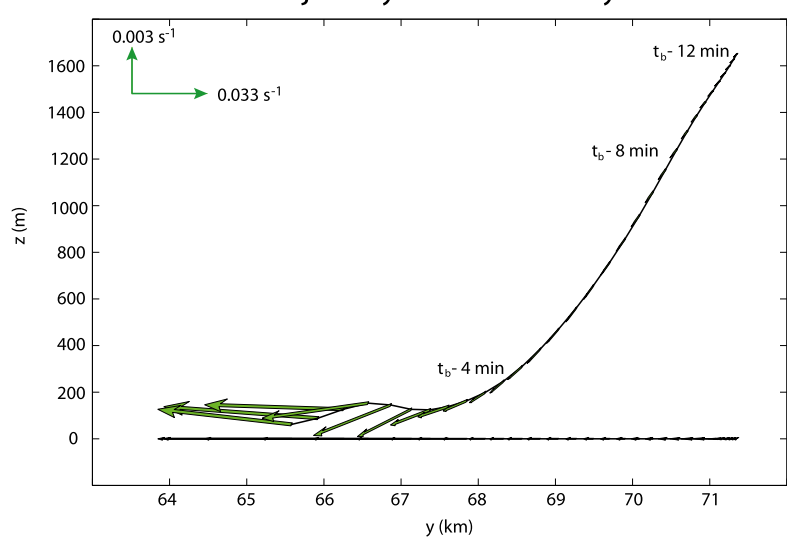

FIG. 7. Total vorticity vector along the trajectory, plotted every $24 \mathrm{~s}$ between 4200 and $4920 \mathrm{~s}$. (a) 3D rendering, looking to the northeast. The horizontal projection of the trajectory and the vorticity vectors is shown in black. (b) Vertical cross section, looking to the west. The time at which the parcel leaves the domain bottom is denoted by $t_{b}$.

passes through the eastern periphery of a region of strong negative buoyancy (Fig. 10, trajectory A), where it is exposed to eastward and then southward baroclinic production. Second, the baroclinic vorticity is also reoriented barotropically, irrespective of additional baroclinic generation. Figure 11 shows the horizontal projection of the parcel stencil before (Fig. 11a) and after (Fig. 11b) the trajectory's eastward turn. While initially the parcels are aligned roughly orthogonally, the stencil is deformed after the bend. The vector is thus rotated clockwise and elongated. The reason for the deformation is that the turn proceeds in a (very nearly) irrotational fashion, such that the cyclonic trajectory curvature is balanced by anticyclonic shear, which deforms the stencil. ${ }^{10}$ At the same time, the change of the trajectory's direction helps align

\footnotetext{
${ }^{10}$ The curve of the trajectory is irrotational because the perturbation pressure gradient force associated with splat and buoyancy forcing at the downdraft base is very nearly irrotational.
}

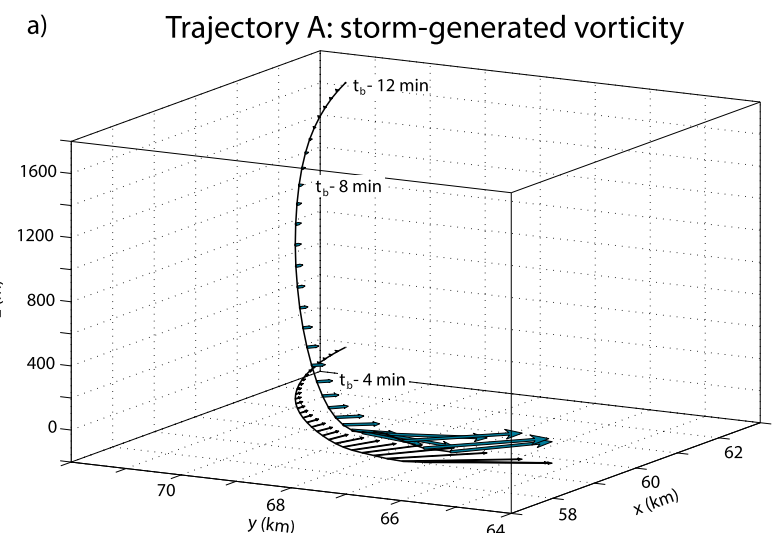

b)

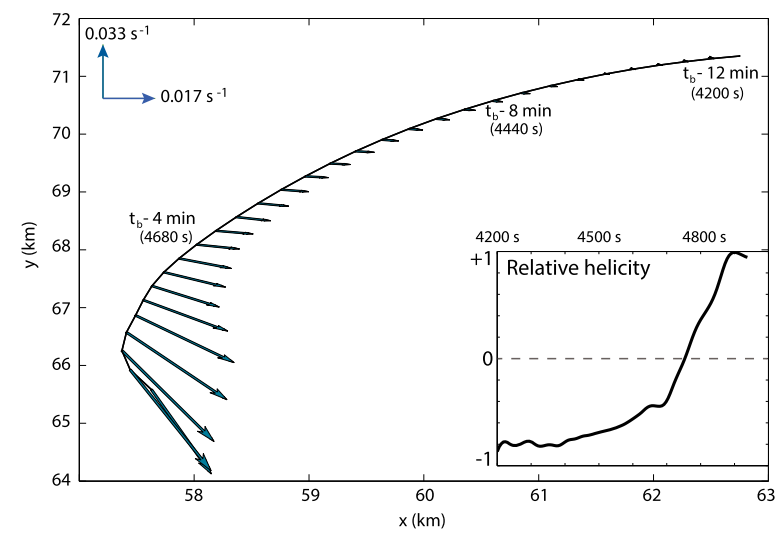

FIG. 8. The storm-generated vorticity vector is plotted along the trajectory within set A every 24 s between 4200 and 4920 s. (a) 3D rendering, looking to the northeast. The horizontal projection of the trajectory and the vorticity vectors is shown in black. (b) Horizontal cross section, highlighting the initial antistreamwise baroclinic vorticity. The inset shows the cosine of the inclination angle between the horizontal baroclinic vorticity and the horizontal velocity vectors, sometimes referred to as relative helicity (e.g., Markowski and Richardson 2010, p. 228), showing the alignment of the vorticity and velocity vectors toward the end of the analysis period. The time at which the parcel leaves the domain bottom is denoted by $t_{b}$.

the local horizontal velocity with the horizontal vorticity. This alignment of initially crosswise vorticity with the velocity vector is often referred to as the "river bend effect" (Davies-Jones et al. 2001, their Fig. 5.15; see also the inset of Fig. 8b).

More interestingly, the baroclinic vorticity vector attains an upward component during the final $90 \mathrm{~s}$ of its descent. Figure 12 reveals the details of this process. As no appreciable baroclinic generation is occurring at this point, the baroclinic vorticity practically remains frozen into the fluid. The key to obtaining cyclonic vertical vorticity is that the parcels at the "tails" of the vorticity vectors descend faster than the parcels at the vectors' "tips." In Fig. 12, this flow structure is visualized by the 

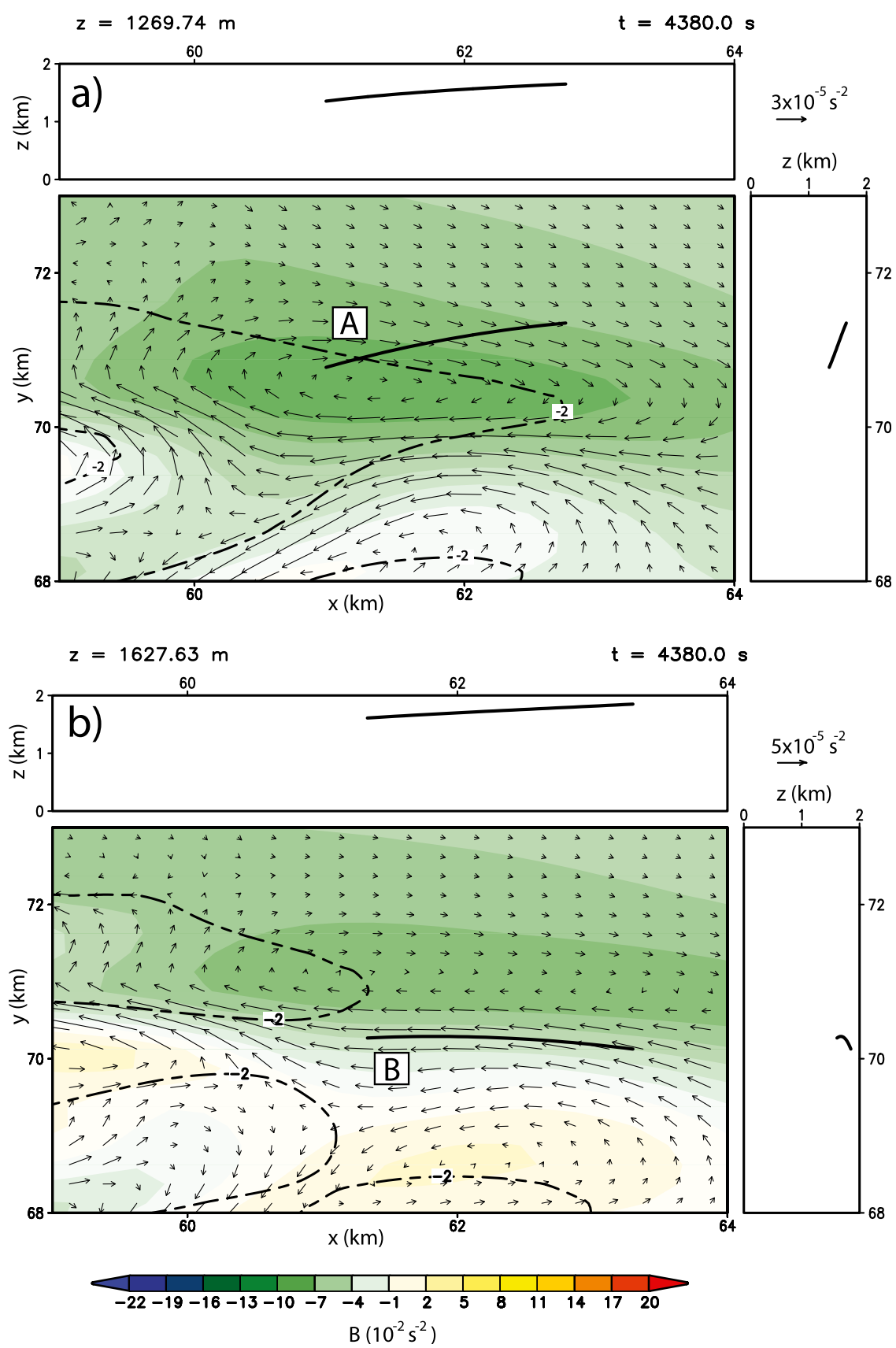

FIG. 9. Buoyancy (shaded) and baroclinic generation (vectors) representative of the environment of trajectories within (a) set A and (b) set B during their early history.

changing tilt of the $a^{2}$-coordinate line as the parcel descends. This is precisely the mechanism proposed by Davies-Jones and Brooks (1993), which will be referred to as the "DJB93 mechanism" below.

The barotropic vorticity for the same parcel with set A is mostly streamwise in the $3 \mathrm{D}$ sense (i.e., the $3 \mathrm{D}$ velocity vector is nearly aligned with the $3 \mathrm{D}$ vorticity vector), as shown in Fig. 13. As the parcel approaches the ground, it is still exposed to the outflow structure that reoriented the baroclinic vorticity, but because its barotropic vorticity is mostly streamwise in the 3D sense, this vorticity becomes nearly horizontal at the end of the trajectory segment (Fig. 13b). This is consistent with the discussion by Davies-Jones and Brooks (1993) who point out that streamwise vorticity (in the 3D sense) becomes purely horizontal as the trajectories bottom out 


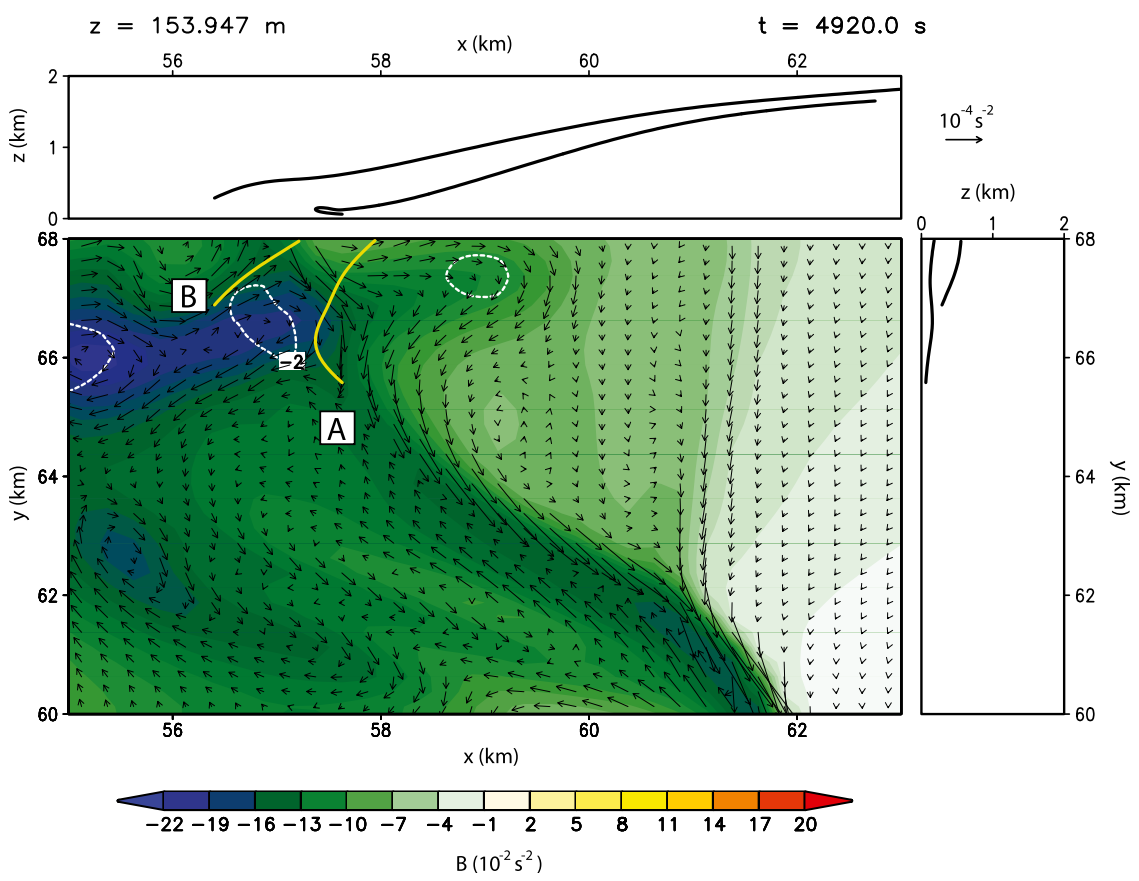

FIG. 10. Horizontal baroclinic-generation vectors and the buoyancy field (shaded) at about $154 \mathrm{~m}$ AGL as well as the downdraft (white dashed line; only the $-2 \mathrm{~m} \mathrm{~s}^{-1}$ contour is shown) at $265 \mathrm{~m}$ AGL. In addition, the two trajectories from sets A and B discussed in the text are shown in yellow for the $(x, y)$ projection and in black for the vertical projections. The buoyancy and vorticity fields vary with height, but they roughly match the height that the trajectories have when reaching the region of strong negative buoyancy.

near the ground, underscoring the requirement for nonstreamwise vorticity (in the $3 \mathrm{D}$ sense) to attain nonzero $\zeta$ at the surface. The result is that the barotropic vorticity vector attains only a comparatively small vertical component as the parcel reaches the domain bottom. Although the magnitude of the barotropic vorticity increases somewhat during the analysis period, the baroclinic vorticity becomes much stronger than the barotropic vorticity, as shown in Fig. 14.

A representative trajectory within set B (e.g., Fig. 3) exhibits the same behavior as the trajectory just presented regarding the barotropic vorticity, which will thus not be shown. However, the early evolution of the baroclinic vorticity is rather different than the previous example, as shown in Fig. 15. Here, the baroclinic production is streamwise initially (at least until $4380 \mathrm{~s}$; Fig. 9b). As the parcel encounters a region of rather strong negative buoyancy at about $4950 \mathrm{~s}$ (trajectory B in Figs. 10 and 15), vigorous eastward baroclinic vorticity production ensues. This renders the horizontal vorticity mostly crosswise, as was the case for the parcel with set A. Subsequent horizontal alignment with the trajectory as well as reorientation into the vertical is achieved as in the previous example (see also the inset of Fig. 15).
The common theme for both sets of trajectories is strong baroclinic generation within a few hundred meters above the ground, a conversion of horizontal vorticity from crosswise to streamwise, and the action of the DJB93 mechanism that yields vertical baroclinic vorticity. This general behavior is also shown when taking the average over all trajectories. Prior to averaging, the trajectories' times were transformed, such that all trajectories reached the domain bottom at the same time. This transformation accounts for the fact that at a given instant, each parcel is in a different phase regarding its height and vorticity changes (some parcels may still be in the forward flank, while others are already descending through the lowest grid level). As seen in Fig. 16, the average parcel behaves very similarly to the two examples just shown. The magnitude of the baroclinic vorticity, as well as its vertical component, is larger than for the barotropic vorticity. Also, as shown in Fig. 17, the average parcel acquires its cyclonic vorticity close to the ground during descent.

To gain a more detailed picture of the roles of baroclinic and barotropic vorticity, Fig. 18 shows the distributions of the total, baroclinic, and barotropic vertical vorticity for all 272 parcels. The values were taken at the point where the parcels descend through the bottom of the scalar domain. Consistent with the previous analysis, 
Trajectory A: baroclinic vorticity and Lagrangian stencil
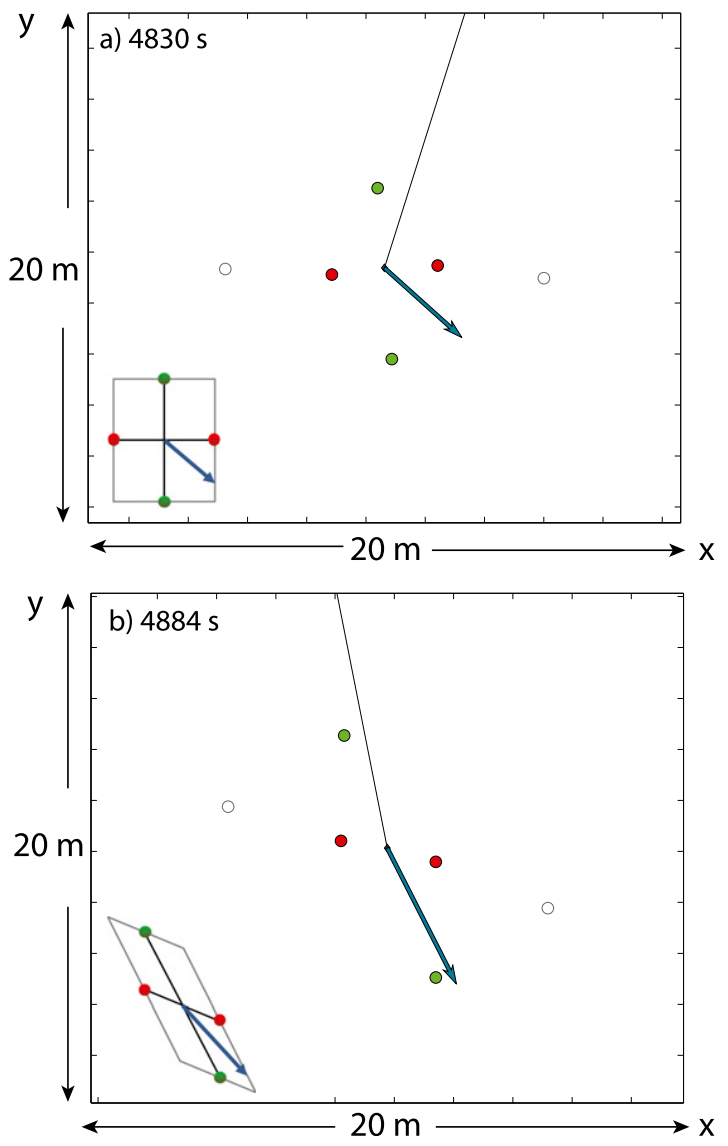

FIG. 11. Horizontal projection of the coordinate stencil (a) before and (b) after the parcels turn southward at the downdraft bottom. The red parcels represent the coordinate line $a^{1}$, the green ones represent $a^{2}$, and the white ones represent $a^{3}$. The blue arrow is the horizontal baroclinic vorticity and the thin black line is the parcel trace. The insets at the bottom-left corners demonstrate how the vector would behave if it was perfectly frozen into the $2 \mathrm{D}$ coordinate stencil (the deviations from this behavior in the actual stencil are due to $3 \mathrm{D}$ effects and baroclinic vorticity production).

the vertical baroclinic vorticity component dominates the vertical barotropic component. The median barotropic vorticity is positive, consistent with Fig. 17, thus slightly reinforcing the baroclinic vertical vorticity. However, whereas the baroclinic vertical vorticity ends up being positive for almost all trajectories, the barotropic vertical component is negative for about $25 \%$ of the trajectories (Fig. 18).

The trajectories corresponding to the outliers (red crosses in Fig. 18) originate within a pocket where the orientation of the vorticity vector is slightly perturbed relative to the base state. This initial crosswise vorticity is reoriented rather strongly, resulting in appreciable vertical barotropic vorticity in the end. However, these parcels do not represent the ambient vorticity very well; their initial vorticity is already contaminated by what
Trajectory A: baroclinic vorticity

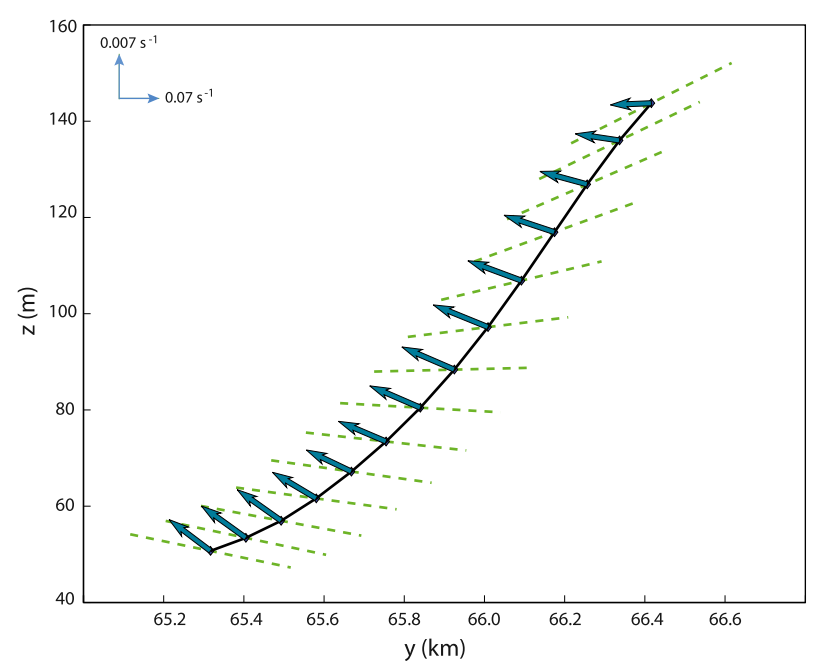

FIG. 12. Trajectory segment (black line) covering the last $72 \mathrm{~s}$ before the parcel reaches the lowest model level. During this interval, the baroclinic vorticity vector (blue arrows) attains an upward component. The green dashed lines represent the orientation of the Lagrangian $a^{2}$ axis. The other coordinate axes are suppressed as they are barely reoriented during the process.

likely are baroclinic effects (these parcels could have been eliminated from the analysis by using different thresholds when identifying suitable parcels, but this would have reduced the overall number of parcels disproportionately).

To check that the evaluation of barotropic vorticity using Cauchy's formula is accurate, we also integrated the barotropic forcing of the vorticity equation along the trajectories using a second-order Runge-Kutta scheme with an integration step of $0.01 \mathrm{~s}$ while updating the forcing every $6 \mathrm{~s}$. The results are nearly indistinguishable from the results using Cauchy's formula and are thus not shown.

\section{Discussion}

An interesting question is how the above analysis relates to tornadogenesis. As discussed in section 3b, we have rather limited faith in the treatment of near-ground trajectories once they descend below the bottom scalar model level, so we cannot describe vortex genesis faithfully. However, when animated, the vertical vorticity field at the lowest scalar model level clearly shows how the rivers and lobes of positive $\zeta$ move downstream and feed into the developing vortex (see also Fig. 5), perhaps suggesting that these extrema are advected into the vortex. In fact, we have not identified suitable vertical velocities that would be consistent with propagation of these extrema via stretching, tilting, or vertical advection. This suggests that a parcel that is part of the $\zeta$ 


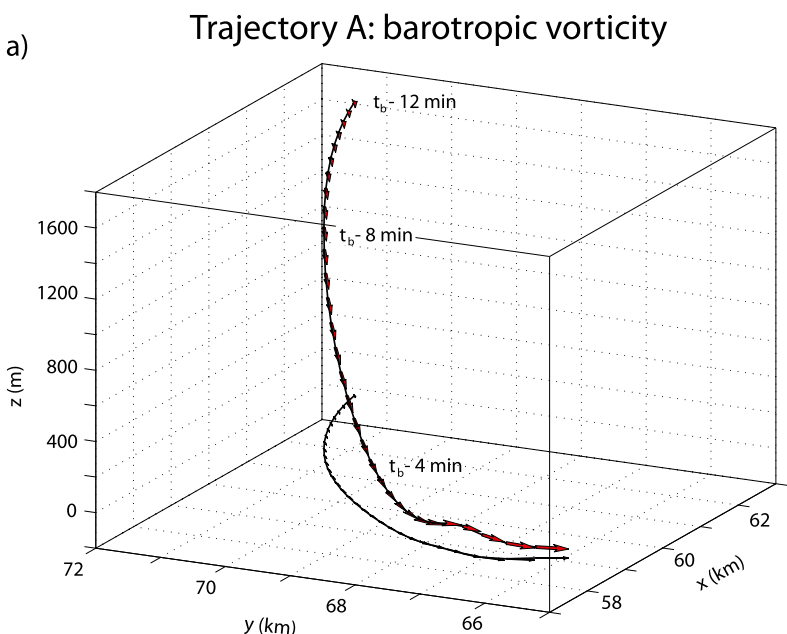

b)

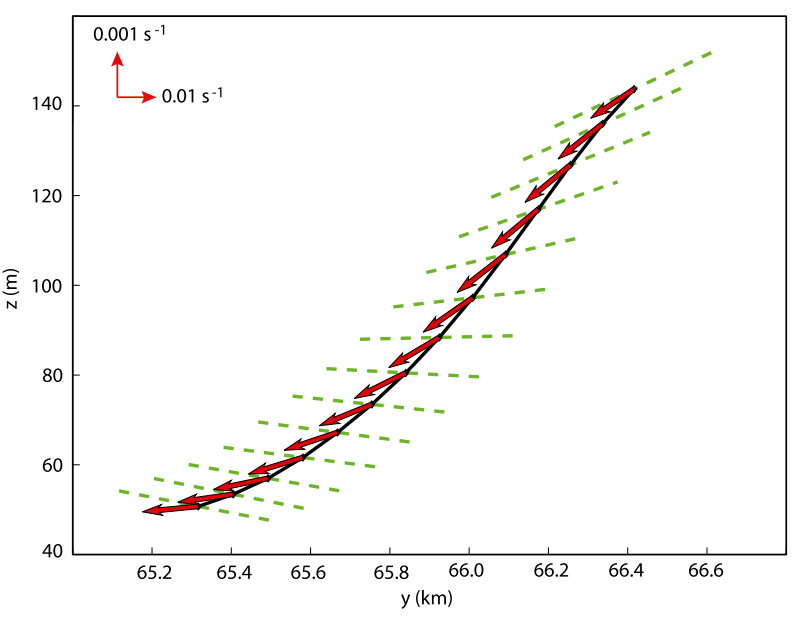

FIG. 13. (a) As in Fig. 7a, but for barotropic vorticity. (b) As in Fig. 12 , but for the barotropic vorticity.

extremum at the foot of the downdraft will remain part of this extremum as it is concentrated by horizontal convergence, even as the parcels descend below $50 \mathrm{~m}$ AGL. Although it could not be demonstrated herein, it thus seems plausible that it is parcels such as these that contribute to an intense near-ground vortex, because they have positive $\zeta$ practically at ground level that is easily concentrated by horizontal convergence provided along the edges of outflow surges. This may be difficult to see in trajectory analyses, for reasons discussed in appendix B.

All forward trajectories analyzed in this and many other simulations carried out in the course of this study reveal that the developing near-ground vortex is fed exclusively by outflow air that has a history of descent, consistent with previous analyses [e.g., as reviewed by Davies-Jones et al. (2001)]. The $\zeta$ streamers (Fig. 5) are associated with surges of outflow (Figs. 4 and 5) emanating from intense downdrafts. The leading edges
Trajectory A: Baroclinic (blue) and barotropic (red) vorticity

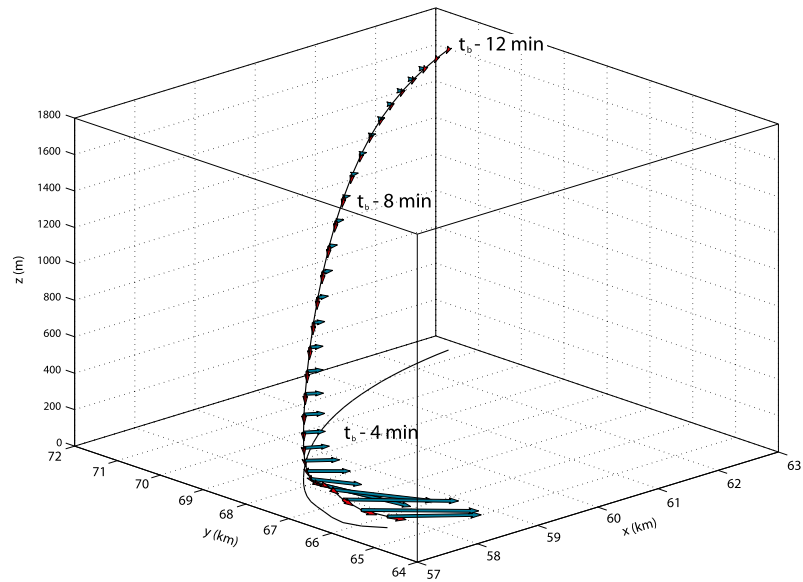

FIG. 14. As in Fig. 7a, but for baroclinic (blue) and barotropic (red) vorticity vectors along the trajectory.

of these horizontal momentum surges are often referred to as secondary rear-flank gust fronts (SRFGF), which are known to accompany the intensification of nearground vertical vorticity also in observed storms (Marquis et al. 2008; Kosiba et al. 2013).

Adlerman et al. (1999) also identified outflow trajectories in their Del City simulation (in addition to trajectories originating east of the rear-flank gust front, which were considered error prone; see also Dahl et al. 2012). Given finer grid spacing, a different numerical model, and an earlier analysis time than used by Adlerman et al. (1999), the downdraft in the present simulation is located more to the north of the updraft than in their analysis, leading to a slightly different trajectory topology. However, their analysis also identified trajectories that gain positive $\zeta$ during descent and exhibit a conversion from crosswise to streamwise vorticity as they interact with the downdraft.

Although a large streamwise vorticity component is beneficial for supercell intensity and longevity (e.g., Bunkers et al. 2006), the development of surface vorticity requires a crosswise vorticity component (e.g., DaviesJones et al. 2001). Otherwise, the initially horizontalvorticity vectors could not approach the ground "tail first" (the parcels located at the tail of the vorticity vector must descend faster than the parcels at its tip to obtain cyclonic vorticity at the ground). This crosswise component may exist in the horizontal plane where downdraft gradients in the cross-flow horizontal direction will facilitate vertical vorticity at the ground [Fig. 9a in Davies-Jones and Brooks (1993)]. The perhaps more interesting scenario involves vorticity being streamwise horizontally but having a crosswise component in the vertical plane. This typically happens if trajectories have 
a) Trajectory B: baroclinic vorticity

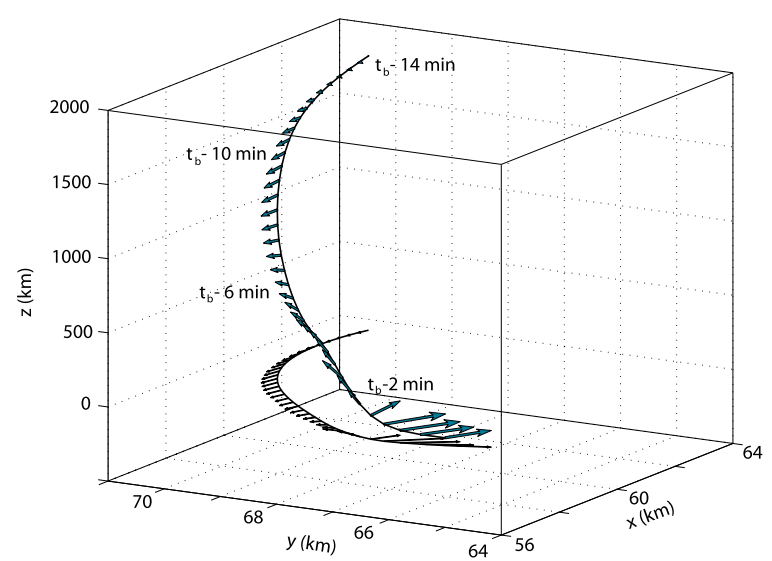

b)

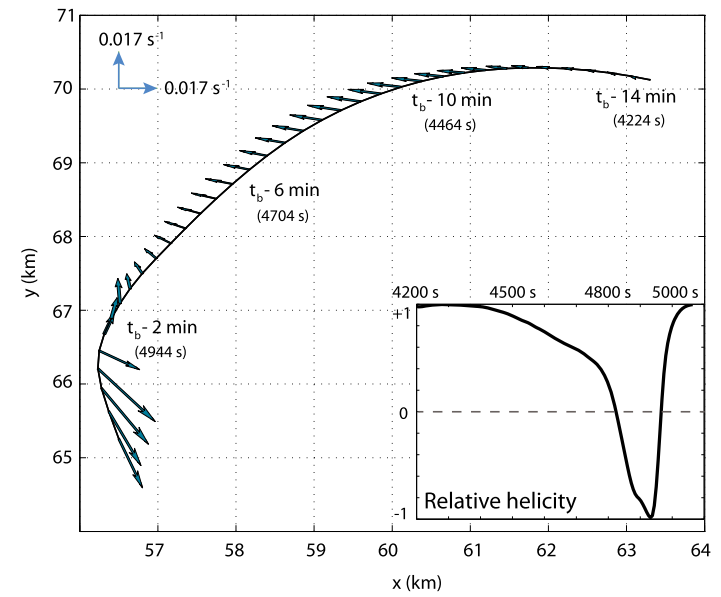

FIG. 15. As in Fig. 8, but for the parcel within trajectory set B. The trajectory is shown from $4200 \mathrm{~s}$ until it descends through the bottom of the scalar model domain at $5064 \mathrm{~s}$, with the vectors plotted every $24 \mathrm{~s}$. (b) Inset shows the cosine of the inclination angle between the horizontal baroclinic vorticity and the horizontal velocity vectors [sometimes referred to as relative helicity (e.g., Markowski and Richardson 2010, p. 228)], showing the alignment of the vorticity and velocity vectors toward the end of the analysis period.

a downward component in the presence of horizontal buoyant production of vorticity. Reorientation of the horizontal vorticity into the vertical is then a result of the lower boundary that forces the trajectories to turn horizontally (Fig. 12).

Perhaps not surprisingly, both baroclinic and barotropic processes act at the same time in a sheared downdraft that is driven by negative buoyancy. ${ }^{11}$ In real-world supercells, where there are precipitation-driven downdrafts that are embedded in strong shear, baroclinic generation

\footnotetext{
${ }^{11}$ Even if the temperature deficit in the downdraft may be small in some cases, the negative buoyancy may be substantial owing to precipitation loading.
}

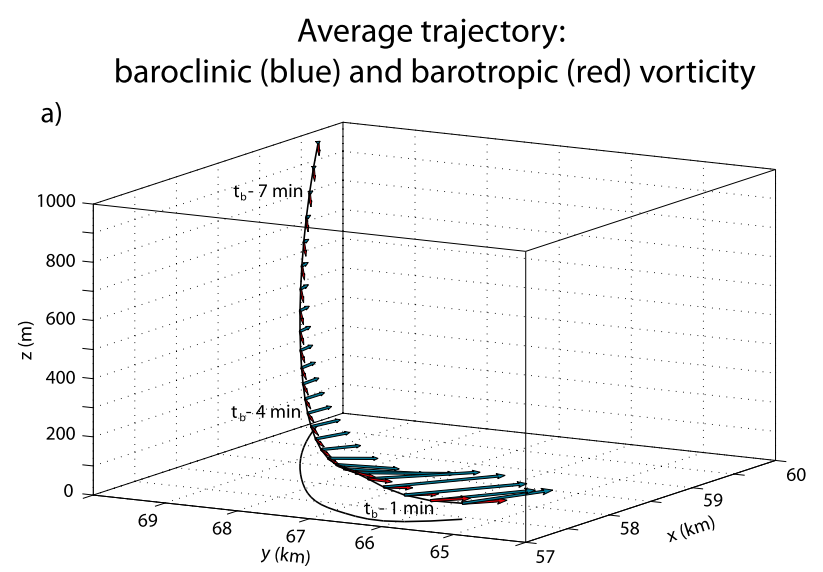

b)

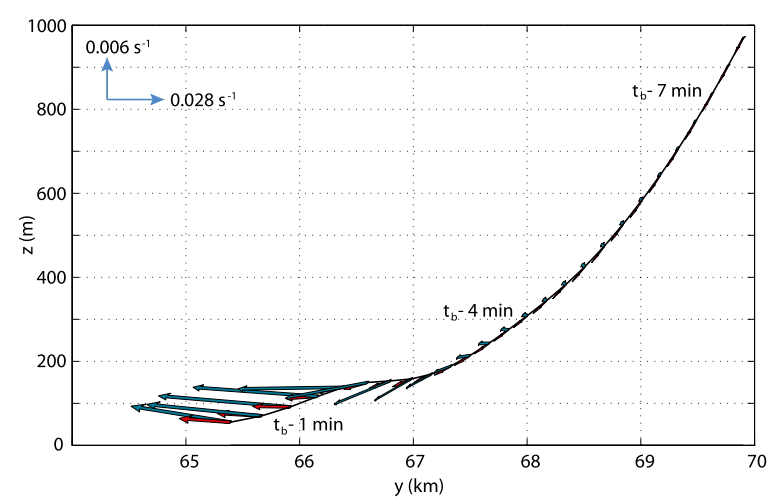

FIG. 16. As in Fig. 14, but for the average over all 272 trajectories, covering $456 \mathrm{~s}$ prior to reaching the bottom of the domain. The early history of the trajectory is not shown for better visibility. No important information is lost because the baroclinic vorticity in the early history is rather small. Vectors are plotted every $18 \mathrm{~s}$.

inevitably occurs in addition to barotropic rearrangement of ambient vorticity vectors. The question merely is how the baroclinic and barotropic vorticity parts will be oriented near the ground. For example, some base-state environments favor substantial crosswise storm-relative vorticity at the height where the parcels originate from, which would likely alter the contribution of barotropic vorticity to near-ground rotation compared to the present simulation. Also, it is conceivable that in some situations there may be substantial cancellation between barotropic and baroclinic vertical vorticity.

Some parcels exited the domain bottom with slightly negative vertical barotropic vorticity (e.g., Fig. 13b). However, the configuration of the stencils could certainly still change in nature at altitudes that are below the analysis grid (i.e., at a height where the trajectories become truly horizontal). It thus seems possible that the distributions in Fig. 18 might change slightly if the parcels were analyzed within the remaining distance to the surface vortex. As mentioned earlier, however, such 


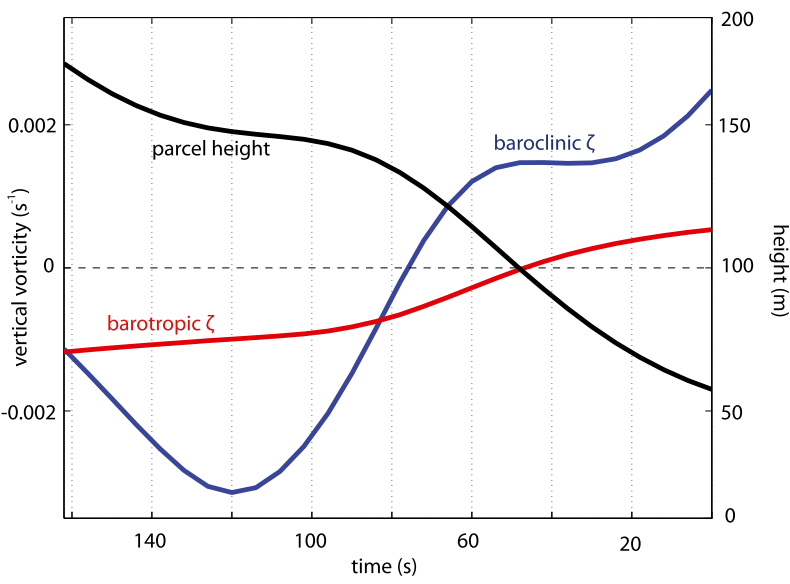

FIG. 17. Time series of the averages of all 272 parcels shown in Fig. 16: height (black line), baroclinic vertical vorticity (blue line), and barotropic vorticity (red line), while positive vertical vorticity is attained. The abscissa represents the time until the parcels reach the lowest scalar model level. Positive vertical vorticity is attained during the last $70 \mathrm{~s}$ of the descent.

a process cannot be confidently depicted in the model once the parcels descend below the bottom scalar level.

An interesting observation is that prior to vortex genesis (from a Lagrangian perspective), the vertical vorticity resulting from the combination of baroclinic and barotropic mechanisms is manifest as shear ( $\zeta$ rivers in Fig. 5). Only farther downstream does the vorticity sheet collapse into a coherent vortex, consistent with the analysis by Gaudet et al. (2006). A possible "shear instability" mechanism for vortex genesis thus is not inconsistent with the baroclinic mechanism involving $\zeta$ production in downdrafts that we find here.

In this study, we did not separate baroclinic vorticity from vorticity imparted to the parcels via diffusion, which might be an important contributor for some parcels in the simulation (there is artificial and numerical diffusion as well as parameterized subgrid-scale mixing in the model). However, since for diffusion to increase a parcel's vorticity, there needs to be large preexisting vorticity nearby (and gradients thereof), diffusion is a secondary effect requiring a nondiffusive mechanism to produce a vorticity extremum in the first place. We are thus confident that the genesis of large surface vorticity is dominated by the baroclinic mechanism described above (and subsequent vertical stretching).

Our results are based on a free-slip simulation; that is, surface friction was neglected, thereby ignoring a potentially important source of near-surface horizontal vorticity (Schenkman et al. 2014). However, the basic mechanisms described herein would not be rendered invalid if an additional source of near-ground horizontal vorticity was added. Including surface friction would

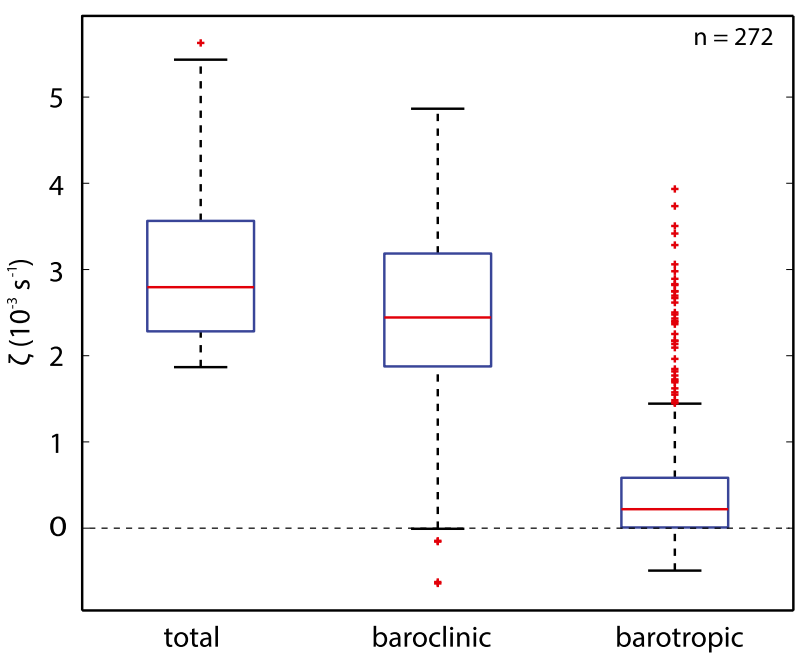

FIG. 18. Box-and-whisker plot showing the distribution of the total, barotropic, and baroclinic vertical vorticity as the parcels reach the domain bottom. The bottom and top of the boxes represent the first and third quartiles, respectively, and the red line is the median of the distributions. Data are treated as outliers (red crosses) if they are above $q_{3}+1.5 \mathrm{IQR}$ or below $q_{1}-1.5 \mathrm{IQR}$, where $q_{1}$ and $q_{3}$ are the first and third quartiles, respectively, and IQR is the interquartile range. The number of parcels in each distribution is 272 and the number of outliers in the distribution of the barotropic vorticity is 40 .

also further complicate the treatment of parcel velocities below the bottom scalar model level, although it would at least provide a consistent dynamical guideline.

\section{Conclusions}

In this study, a decomposition of vorticity into barotropic and nonbarotropic parts was used to separate the roles of ambient and storm-generated vorticity in nearground rotation. This formalism was applied to a supercell, which was simulated with the Bryan Cloud Model (CM1) and initialized within the canonical Del City base state. Out of several million forward trajectories, a few hundred were identified that contribute to near-ground vertical vorticity at the base of downdrafts.

Our primary results may be summarized as follows:

- We use a Lagrangian framework to analyze the barotropic vorticity evolution by tracking deforming volume elements into which the barotropic vorticity vector is embedded. The barotropic vorticity components were obtained in Lagrangian coordinates and mapped to Cartesian coordinates using Cauchy's formula. The nonbarotropic vorticity was treated as the residual between the known total vorticity along the trajectories and the barotropic vorticity.

- The development of near-ground rotation is an outflow phenomenon; that is, rotation develops in cool air 


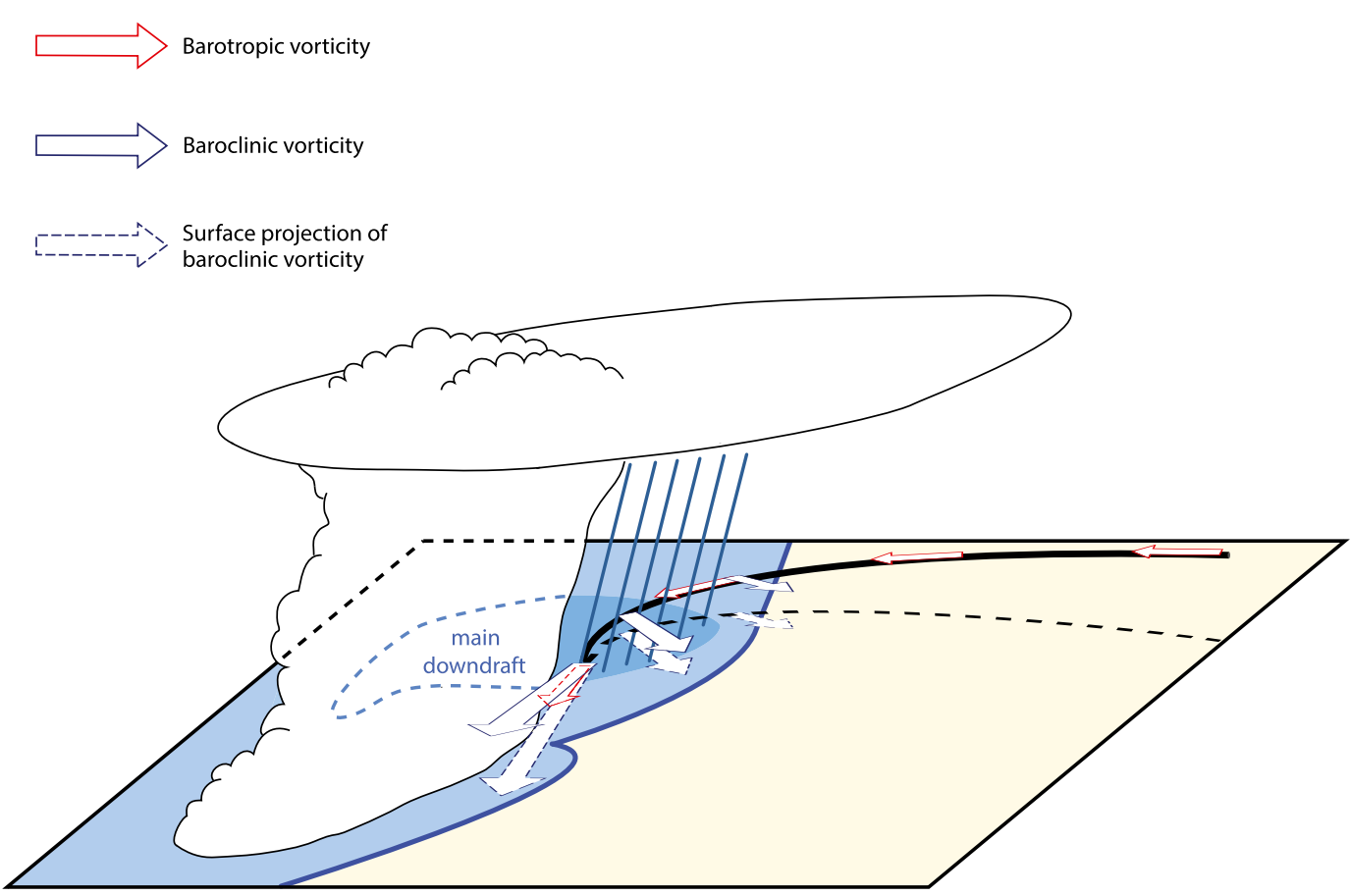

FIG. 19. Conceptual model highlighting the evolution of barotropic vorticity (red arrows) and baroclinic vorticity (blue arrows) along a trajectory (black line) consistent with the simulation. The dashed black line is the surface projection of the trajectory. The early, weak baroclinic production of horizontal vorticity is suppressed in this picture, and only the effect of the main downdraft is shown.

behind the gust front. Numerous vertical vorticity extrema develop within the cold pool at the lowest model level, while in the inflow region, the vertical vorticity remains practically zero at that level.

- There are distinct rivers of cyclonic (and anticyclonic) shear vorticity that originate from the base of downdrafts. Near-ground tornado-like vortices are fed by such cyclonic $\zeta$ rivers during their genesis.

- Early in the history of the analyzed trajectories, some parcels gain antistreamwise horizontal baroclinic vorticity, while others gain crosswise horizontal baroclinic vorticity. A few hundred meters above the ground, however, strong baroclinic generation yields large crosswise horizontal vorticity for all trajectories. Subsequent alignment of the baroclinic vorticity with the velocity vector in the horizontal plane is achieved by additional (streamwise) baroclinic generation and the river bend effect. The horizontal vorticity thus becomes streamwise for all analyzed trajectories, irrespective of the orientation of the baroclinic vorticity in the earlier history.

- The key for the development of vertical positive vorticity near the ground is that horizontal vorticity vectors (either generated baroclinically, or imported from the environment) descend tail first (i.e., the tails of the vorticity vectors descend faster than their tips, and vice versa for negative near-ground vertical vorticity). This can only be achieved if the vorticity has a nonstreamwise component in either the horizontal or vertical plane.

- In this simulation, the nonstreamwise component arises from horizontal baroclinic vorticity generation while the parcel trajectories are sloped downward, resulting in crosswise vorticity in the vertical plane, although the horizontal vorticity becomes largely streamwise. Since the downdraft decreases along the direction of the vorticity vector owing to the lower boundary, this vector is reoriented into the vertical during descent (descending tail first). This is the DJB93 mechanism.

- The initially horizontal vorticity imported from the environment, herein represented by the barotropic vorticity, remains nearly aligned with the $3 \mathrm{D}$ velocity vector within the analysis period. Consequently, the vertical component of the barotropic vorticity near the ground remains rather small. Thus, in the present simulation, the generation of surface vorticity is dominated by baroclinic effects.

The conceptual model shown in Fig. 19 summarizes the main processes that lead to near-ground vertical vorticity. Future work is targeted toward quantifying the relative roles of baroclinic and barotropic vorticity in environments in which parcels exhibit a larger horizontal crosswise vorticity component initially than the present simulation. 
Also, we intend to explicitly include baroclinic and frictional generation in the Lagrangian framework.

Acknowledgments. George Bryan is gratefully acknowledged for providing and supporting the CM1 model. Also, we greatly benefited from discussions over the recent years with Paul Markowski, George Bryan, Bob Davies-Jones, Chuck Doswell, Craig Epifanio, and Daniel Dawson. Comments on the manuscript by the Convective Storms Group (Brice Coffer, Jason Davis, Christopher MacIntosh, and Keith Sherburn), Yvette Richardson, Richard Rotunno, and two anonymous reviewers are greatly appreciated. This work is supported by the National Science Foundation under Grants ATM-0758509 and AGS-1156123.

\section{APPENDIX A}

\section{Cauchy's Formula and the Barotropic Vorticity}

In this appendix we demonstrate how Cauchy's vorticity formula may be obtained in a straightforward manner by considering the behavior of a material line segment. Moreover, we demonstrate that this formula may be interpreted as a passive transformation of the barotropic vorticity components relative to the covariant Lagrangian basis to a Cartesian basis at time $t$, as discussed in section 2a. This interpretation allows us to infer the barotropic vorticity components relative to the (covariant) Lagrangian basis. For alternative derivations and interpretations of Cauchy's formula the reader is referred to, for example, Dutton (1976), Epifanio and Durran (2002), or Davies-Jones (2006).

Let $\left(r^{1}, r^{2}, r^{3}\right)$ represent fixed Cartesian coordinates, and let $\left(a^{1}, a^{2}, a^{3}\right)$ represent the material (Lagrangian) coordinates as discussed in section $2 \mathrm{a}$. The components of a material line segment at an arbitrary time $t$ may be represented relative to the covariant basis of the deformed Lagrangian coordinates or relative to the Cartesian basis. With respect to the covariant Lagrangian basis, the line segment is time independent, and its Cartesian coordinates are obtained via the coordinate differential

$$
d r^{i}=\frac{\partial r^{i}}{\partial a^{\nu}} d a^{\nu}
$$

where, again, Einstein's summation convention is in force.

The time rate of change of the material line segment $d r^{i}$ is given by (see also Batchelor 2002, p. 132)

$$
\frac{d\left(d r^{i}\right)}{d t}=\frac{d}{d t}\left[\frac{\partial r^{i}}{\partial a^{\nu}} d a^{\nu}\right]=\frac{\partial u^{i}}{\partial a^{\nu}} d a^{\nu}
$$

$$
=\frac{\partial u^{i}}{\partial r^{k}} \frac{\partial r^{k}}{\partial a^{\nu}} d a^{\nu}=\frac{\partial u^{i}}{\partial r^{k}} d r^{k},
$$

where $u^{i}=u_{\mathbf{a}}^{i}(t)$ represents the parcel's velocity and $d / d t$ is the Lagrangian time derivative operator. This equation bears much resemblance with the vorticity equation in inviscid, barotropic flow,

$$
\frac{d}{d t}\left[\frac{\omega^{i}}{\rho}\right]=\frac{\partial u^{i}}{\partial r^{k}}\left[\frac{\omega^{k}}{\rho}\right] .
$$

Indeed, these equations are identical if we set $d r^{i}=\alpha\left(\omega^{i} / \rho\right)$, which is a valid choice since Eq. (A1) is true for arbitrary $d r^{i}$. Here, $\alpha$ is a proportionality constant accounting for the different units of $d r^{i}$ and $\omega^{i} / \rho$. In Eq. (A4), $\rho=\rho_{\mathbf{a}}(t)$ and $\omega^{i}=\omega_{\mathbf{a}}^{i}(t)$ are the density and vorticity, respectively, following a parcel. The righthand side of Eq. (A4) contains the stretching and reorientation of vortex lines (e.g., Kundu and Cohen 2008, p. 155). This demonstrates that the vector $\omega^{i} / \rho$ and the associated vortex-line segment behave like material line segments. We thus substitute $d r^{i}=\alpha\left[\omega^{i}(t) / \rho(t)\right]$ and $d a^{\mu}=\alpha\left[\omega_{0}^{\mu} / \rho\left(t_{0}\right)\right]$ into Eq. (A1), which results in

$$
\frac{\omega^{i}(t)}{\rho(t)}=\frac{\partial r^{i}}{\partial a^{\nu}}\left[\frac{\omega_{0}^{\nu}}{\rho\left(t_{0}\right)}\right] ;
$$

see also Saffman (1992). This is Cauchy's formula, which we may write as

$$
\begin{aligned}
\omega^{i}(t) & =\frac{\partial r^{i}}{\partial a^{\nu}}\left[\frac{\rho(t)}{\rho\left(t_{0}\right)} \omega_{0}^{\nu}\right] \\
& =\frac{\partial r^{i}}{\partial a^{\nu}}\left[\left(J_{\mathbf{a}}^{\mathbf{r}}\right)^{-1} \omega_{0}^{\nu}\right],
\end{aligned}
$$

where $\rho\left(t_{0}\right) / \rho(t)=J_{\mathbf{a}}^{\mathbf{r}}\left(\right.$ e.g., Aris 1962, p. 83) and $J_{\mathbf{a}}^{\mathbf{r}}$ is the Jacobian of the transformation from the covariant Lagrangian basis to the Cartesian basis.

To justify that this equation may be interpreted as a passive transformation from the covariant Lagrangian basis to the Cartesian basis, we will demonstrate that the (contravariant) components of the vorticity vector obey the tensor transformation law. This way we also obtain the contravariant barotropic vorticity components in Lagrangian coordinates. Physically, fulfillment of the tensor transformation behavior means that the vorticity vector is a geometric object that exists independently of any coordinate systems. To obtain the (contravariant) components of the barotropic vorticity vector in Lagrangian coordinates, we use the transformation properties of the vorticity vector. In general coordinates, its contravariant components are given by (e.g., Dutton 1976, p. 144) 

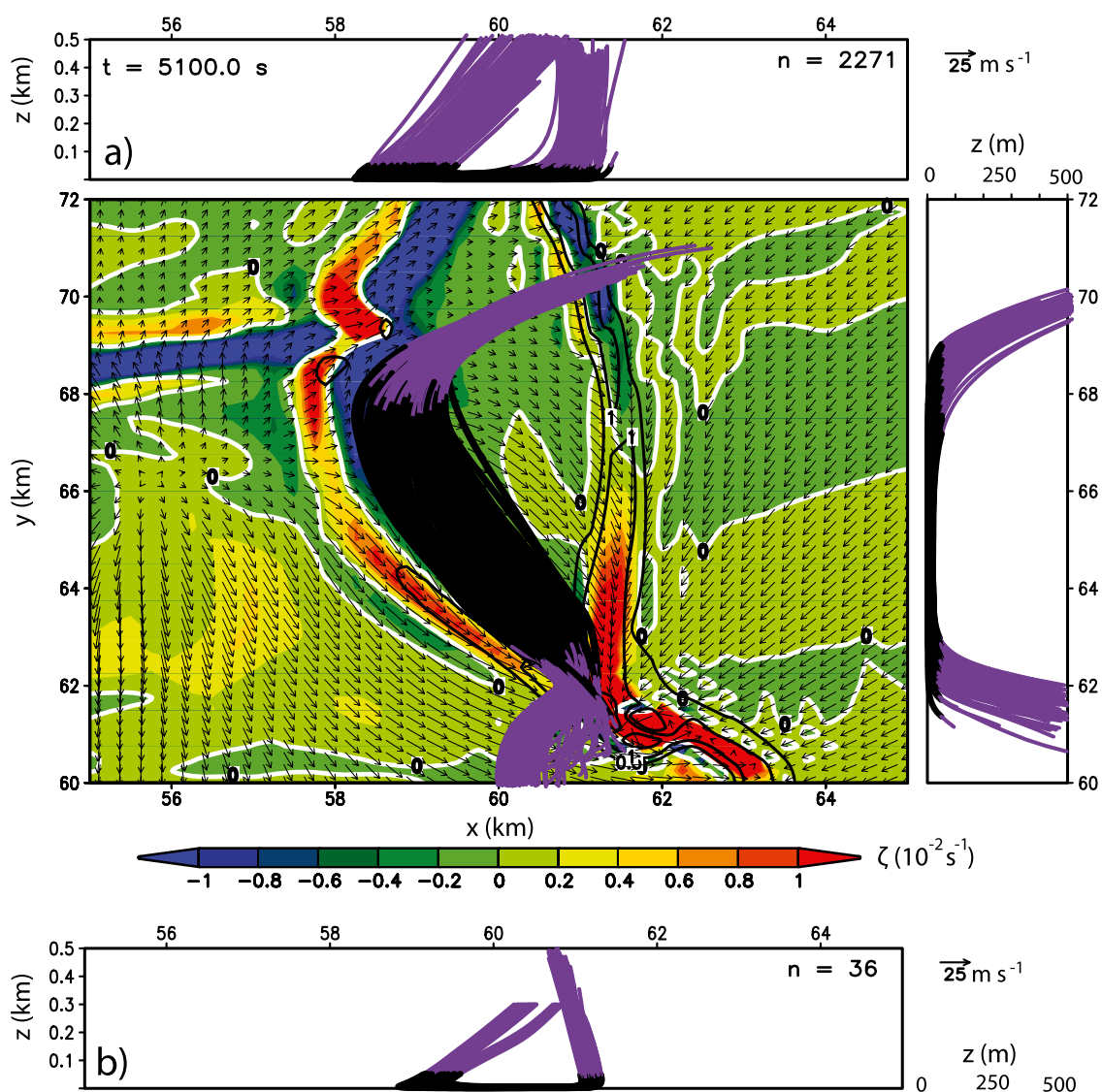

$\overrightarrow{25} \mathrm{~m} \mathrm{~s}^{-1}$
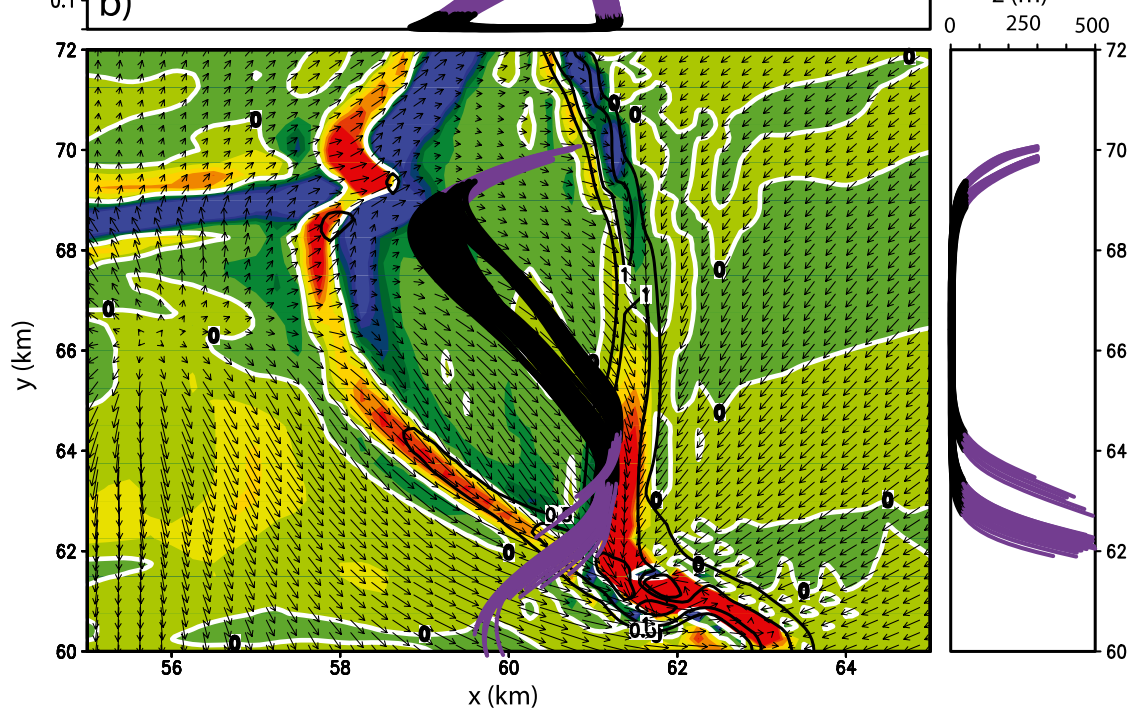

FIG. B1. Trajectories that become part of (a) the developing vortex and (b) the eastern $\zeta$ river. The magenta (black) segments show parcel heights $>50(\leq 50) \mathrm{m}$. Also shown is the horizontal velocity extrapolated to $z=0 \mathrm{~m}$ and the vertical vorticity calculated on the staggered grid at $z=0 \mathrm{~m}$. The black contours denote updraft $\left(0.5\right.$ and $\left.1 \mathrm{~m} \mathrm{~s}^{-1}\right)$ at $50 \mathrm{~m} \mathrm{AGL}$.

$$
\omega^{k}=\frac{\epsilon^{i j k}}{\sqrt{g}} \frac{\partial u_{j}}{\partial r^{i}}
$$

$$
\epsilon^{i j k}=\left\{\begin{array}{lll}
+1 & \text { if } i, j, k \text { are an even permutation of } 1,2,3 \\
-1 & \text { if } i, j, k \text { are an odd permutation of } 1,2,3 \\
0 & \text { if } \quad \text { any two indices are equal, }
\end{array}\right.
$$

where $\epsilon^{i j k}$ is the permutation symbol, 
and $g$ is the determinant of the metric tensor of the $r^{i}$ coordinates. We may again let $r^{i}$ represent the Cartesian grid, where $g=1$. Upon changing the coordinate basis, we obtain (see Aris 1962, p. 145)

$$
\begin{aligned}
\omega^{k} & =\frac{\partial r^{i}}{\partial a^{\alpha}} \frac{\partial r^{j}}{\partial a^{\beta}} \frac{\partial r^{k}}{\partial a^{\gamma}} \frac{\bar{\epsilon}^{\alpha \beta \gamma}}{\sqrt{\bar{g}}} \frac{\partial u_{j}}{\partial r^{i}} \\
& =\frac{\partial r^{i}}{\partial a^{\alpha}} \frac{\partial r^{j}}{\partial a^{\beta}} \frac{\partial r^{k}}{\partial a^{\gamma}} \frac{\partial a^{\mu}}{\partial r^{i}} \frac{\partial a^{\nu}}{\partial r^{j}} \frac{\bar{\epsilon}^{\alpha \beta \gamma}}{\sqrt{g}} \frac{\partial \bar{u}_{\nu}}{\partial a^{\mu}},
\end{aligned}
$$

where the antisymmetric nature of the permutation symbol was used. Here, $\bar{g}$ is the determinant of the metric tensor of the $a^{\mu}$ (=Lagrangian) coordinates, and $\bar{u}_{\mu}$ represents the velocity components in these coordinates. Noting that $\left(\partial r^{i} / \partial a^{\alpha}\right)\left(\partial a^{\mu} / \partial r^{i}\right)=\delta_{\alpha}^{\mu}$, where $\delta_{\alpha}^{\mu}$ is the unit tensor, the above expression simplifies to

$$
\begin{aligned}
\omega^{k} & =\delta_{\alpha}^{\mu} \delta_{\beta}^{\nu} \frac{\partial r^{k}}{\partial a^{\gamma}} \frac{\bar{\epsilon}^{\alpha \beta \gamma}}{\sqrt{\bar{g}}} \frac{\partial \bar{u}_{\nu}}{\partial a^{\mu}} \\
& =\frac{\partial r^{k}}{\partial a^{\gamma}} \frac{\bar{\epsilon}^{\alpha \beta \gamma}}{\sqrt{\bar{g}}} \frac{\partial \bar{u}_{\beta}}{\partial a^{\alpha}} \\
& =\frac{\partial r^{k}}{\partial a^{\gamma}} \Omega^{\gamma} .
\end{aligned}
$$

Here,

$$
\Omega^{\gamma}=\frac{\bar{\epsilon}^{\alpha \beta \gamma}}{\sqrt{g}} \frac{\partial \bar{u}_{\beta}}{\partial a^{\alpha}}
$$

represents the (contravariant) barotropic vorticity components in Lagrangian coordinates and $\sqrt{\bar{g}}=J_{\mathbf{a}}^{\mathbf{r}}=$ $\rho\left(t_{0}\right) / \rho(t)$.

This demonstrates that vorticity [Eq. (A8)] indeed obeys the vector transformation law. The expression (A14) for the parcel's vorticity is not very practical, however, because the velocity components $\bar{u}_{\beta}$ need to be expressed with respect to the nonorthogonal Lagrangian basis. However, using Eq. (A14) and Cauchy's formula [Eq. (A6)] we find that

$$
\omega^{k}=\frac{\partial r^{k}}{\partial a^{\gamma}} \Omega^{\gamma}=\frac{\partial r^{k}}{\partial a^{\nu}}\left[\left(\mathbf{J}_{\mathbf{a}}^{\mathbf{r}}\right)^{-1} \omega_{0}^{\nu}\right]
$$

implying

$$
\begin{aligned}
\Omega^{\nu}(t) & =\left[J_{\mathbf{a}}^{\mathbf{r}}(t)\right]^{-1} \omega_{0}^{\nu} \\
& =\frac{\rho(t)}{\rho\left(t_{0}\right)} \omega_{0}^{\nu},
\end{aligned}
$$

which are the parcel's (contravariant) barotropic vorticity components expressed in Lagrangian coordinates.

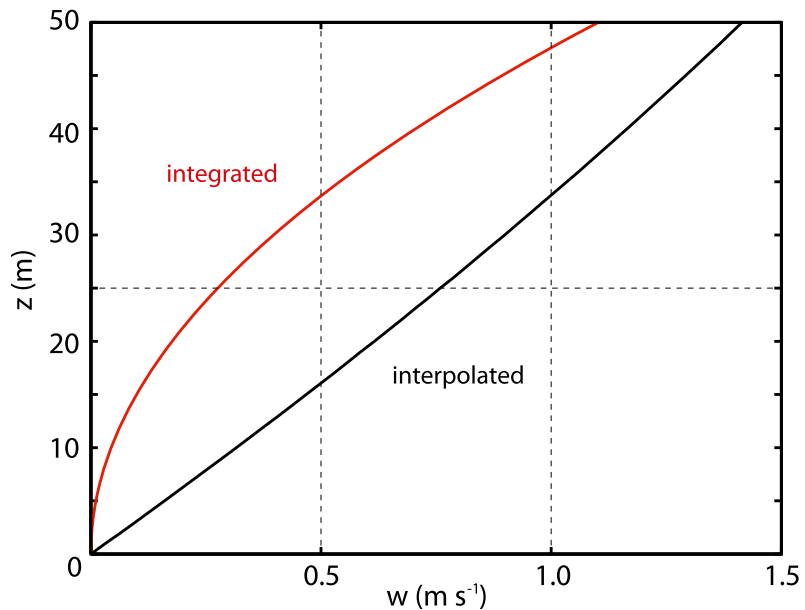

FIG. B2. Vertical profile of the vertical velocity within the western $\zeta$ river as experienced by a parcel in the simulation (polynomial interpolation; black line) and based on integration of the incompressible mass continuity equation as discussed in the text (red line).

As before [Eq. (6)], $\omega_{0}^{\nu} \equiv\left(\boldsymbol{\omega} \cdot \mathbf{g}^{\nu}\right)_{t_{0}}=\boldsymbol{\omega}\left(t_{0}\right) \cdot \mathbf{e}^{\nu}$. The advantage of this formulation is that deformation of the fluid volume is contained in the coordinate system, yielding a rather simple expression for, and interpretation of, the barotropic vorticity components.

This formulation is consistent with the initial condition [Eq. (1)], because $J_{\mathbf{a}}^{\mathbf{r}}=1$ at $t_{0}$, so that initially the barotropic vorticity is given by the total vorticity. Also, the definition correctly reflects the nonconservation of the Lagrangian components of the barotropic vorticity $\Omega^{\nu}$ in compressible flows, as described in section 2 .

Cauchy's formula may thus be interpreted a passive transformation of the contravariant components of the barotropic vorticity from the covariant Lagrangian basis to the Cartesian basis at time $t$. In baroclinic (or viscous) flows, we replace $\omega^{i}$ with $\omega_{\mathrm{BT}}^{i}$ and $\Omega^{\nu}$ with $\Omega_{\mathrm{BT}}^{\nu}$. Our formulation is consistent with the "operator" form of Cauchy's formula [Eq. (4)], where

$$
\Pi_{\cdot \nu}^{i}=\left(J_{\mathbf{a}}^{\mathbf{r}}\right)^{-1} \frac{\partial x^{i}}{\partial a^{\nu}} .
$$

The $\Pi$ operator is the inverse of the so-called Piola transformation (Casey and Naghdi 1991).

\section{APPENDIX B}

\section{Alternative Criteria for Selecting Parcels}

In the main text, we focused on parcels that became part of the "western" $\zeta$ streamer. However, none of these parcels actually entered the near-ground vortex because they quickly rose away from the surface while in the river. The purpose of this appendix is to highlight 


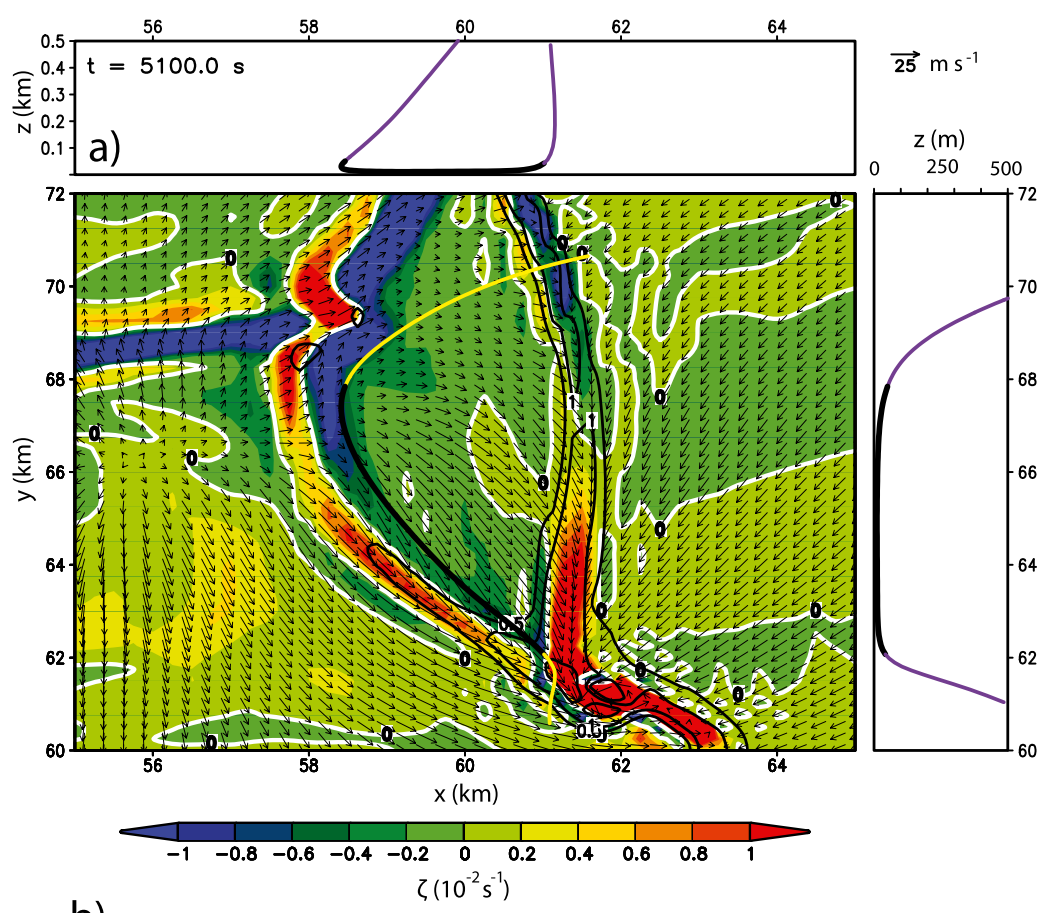

b)

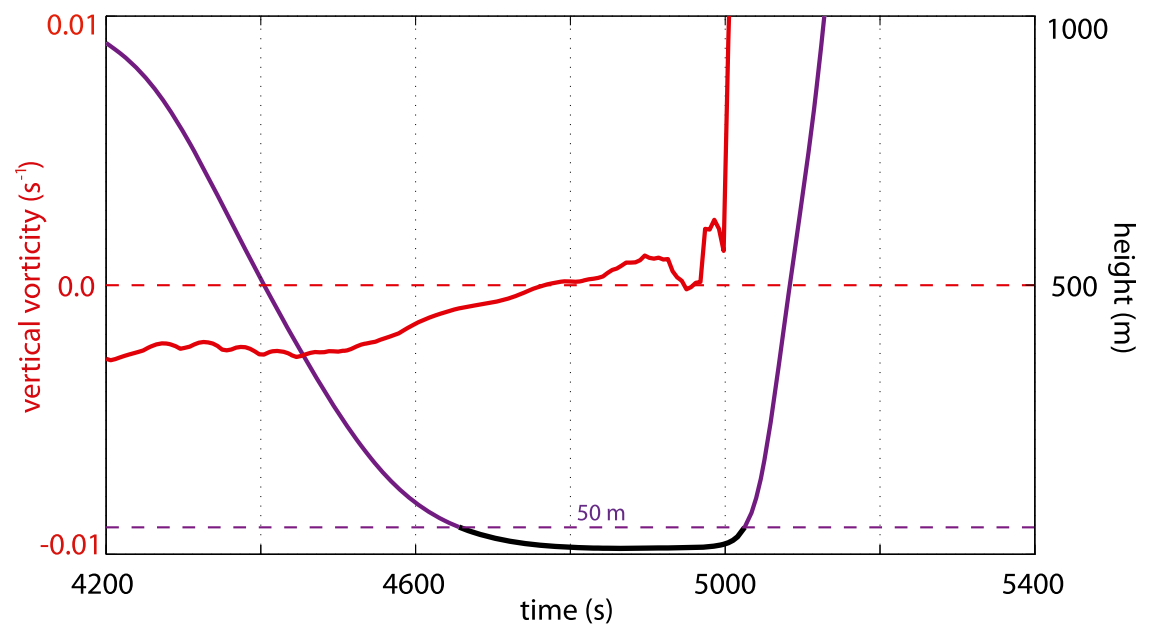

FIG. B3. (a) As in Fig. B1b, but for a representative trajectory that passes through the developing near-ground vortex. For better visibility, the trajectory is colored in yellow for heights above $50 \mathrm{~m}$ AGL. (b) Time evolution of the vertical vorticity (red) and parcel height (magenta for $z>50 \mathrm{~m}$ and black for $z \leq 50 \mathrm{~m}$ ).

some issues with near-ground trajectories and how these might affect the results.

\section{a. Near-ground vertical velocities}

As shown in Fig. B1, the $\zeta$ rivers are also regions of upward motion. This is not surprising, because vertical vorticity is concentrated in regions of horizontal convergence near the edges of the internal surges. A parcel that has gained vertical vorticity close to the ground and that becomes part of a $\zeta$ streamer will unavoidably find itself in a regime of ascent. What determines the strength of the vertical velocity $w$ ? In the model, $w$ below the lowest scalar level is traditionally determined by linear interpolation. In the current implementation, a polynomial interpolator is used, but either way $w$ is determined by the $w$ field above the parcel's location. Physically, however, the vertical velocity is to a good approximation determined by the divergence of the horizontal velocity below the parcel's location and the condition that $w=0$ at $z=0$. Hence, for a given wind profile, neither the linear nor any 
higher-order interpolation scheme guarantees mass conservation. However, even if one chose to integrate the mass continuity equation to obtain $w(z)$, errors may result from the choice of the subdomain horizontal wind profile, as discussed in section $3 \mathrm{~b}$.

As an illustrative but highly simplified example, we considered the vertical velocity profile that a parcel experiences in the simulation amidst the western $\zeta$ streamer, $[(x, y)=(60,63) \mathrm{km}$ at $4800 \mathrm{~s}]$. This vertical velocity profile results from the Lagrangian interpolator. This we compared to a mass continuity-based $w(z)$ profile. Now we assume a horizontal wind profile that decreases linearly to zero toward the ground [i.e., $\mathbf{v}_{h}=(\alpha, \beta) z$, where $\alpha$ and $\beta$ are constants that were adjusted to meet the horizontal velocity at the lowest scalar grid level]. To obtain the $w(z)$ profile, we integrated the incompressible mass continuity equation with respect to $z$. This scenario implies that the horizontal convergence decreases toward the ground, loosely mimicking the real-world behavior. ${ }^{\mathrm{B} 1}$ As shown in Fig. B2, the interpolated vertical velocity increases more rapidly with height near the ground than the mass continuity-based vertical velocity. This simple example shows that the interpolated vertical velocity may significantly differ from a mass continuity-based $w(z)$ profile.

Given that there is no guarantee that the parcels experience physically consistent vertical velocities near the ground, we are thus skeptical about the behavior of the parcels beneath the scalar model domain within the $\zeta$ rivers in our simulation.

\section{b. Parcels in the near-ground vertical-vorticity maximum}

The perhaps most straightforward and most common way of identifying parcels of interest is to look for trajectories along which large $\zeta$ develops close to the ground. Indeed, this procedure yields a large number of parcels in our simulation, some of which are shown in Fig. B1a. One representative parcel is shown in Fig. B3. Upon inspection of its $\zeta$ evolution, the following becomes apparent:

- The parcel acquires positive $\zeta$ very close to the surface ( $\$ 20 \mathrm{~m}$ ), below the physical scalar model domain.

- The tendencies seem unphysical. Most strikingly, the parcel exhibits an oscillation of $\zeta$ prior to reaching the vortex, with $\zeta$ temporarily dropping below zero. This would contradict the notion that parcels need to

\footnotetext{
${ }^{\mathrm{B} 1}$ Little is known about the vertical profile of the horizontal wind within outflow surges, so this example merely highlights that the interpolated $w$ profile is not in general consistent with mass continuity.
}

acquire positive vertical vorticity in downdraft prior to reaching the ground for surface $\zeta$ to increase. We suspect that this erratic vertical vorticity evolution is caused by the poorly constrained lower boundary condition for the horizontal wind, as discussed in section $3 \mathrm{~b}$.

- The vorticity field in the path of these trajectories seems rather noisy (which may also contribute to the $\zeta$ oscillations), as suggested by the small-scale anticyclonic extrema surrounding the vertical-vorticity maximum in, for example, Fig. B3.

- Conspicuously, only those parcels that have not been exposed to an extended period of horizontal convergence (unlike those parcels within the $\zeta$ rivers) enter the near-ground vortex.

As discussed in the previous subsection, there is no guarantee that the vertical velocities of near-ground parcels are physically meaningful, and it is possible that parcels within the $\zeta$ rivers are evacuated upward unphysically quickly. This possibility, along with the poorly constrained horizontal near-ground winds, might explain why none of the parcels entering the near-ground vortex acquire cyclonic vorticity within the downdraft, but only as they enter the vortex.

The reason for these inconsistencies is likely that there currently is no obvious way of relating the (physically consistent) model solution on the grid to the parcels in "no-man's land" beneath the lowest scalar model level.

\section{c. Parcels in the "eastern" $\zeta$ river}

Despite the small initial parcel spacing $(d x=d y=$ $25 \mathrm{~m}, d z=50 \mathrm{~m}$ ), no parcels were found that entered the "eastern" $\zeta$ river at $50 \mathrm{~m}$ AGL, so that we cannot confidently analyze the origin of this river. However, several parcels were found that enter the eastern $\zeta$ streamer very close to the ground (Fig. B1b). These parcels, like the ones in the western $\zeta$ river, experience strong upward velocities prior to arrival at the near-ground vortex. As discussed, this might be an artifact of how the vertical velocity on the grid is mapped to the parcels. We are thus quite skeptical that the ascent of all parcels out of the $\zeta$ rivers prior to reaching the near-ground $\zeta$ maximum reflects the actual simulated flow field realistically.

In summary, we suspect that the trajectory behavior and the $\zeta$ tendencies of the parcels are rather sensitive to the treatment of the 3D velocity vector near the ground and possibly to noise in the vorticity field. Ongoing research is focused on these sensitivities as well as on possible remedies for inaccurate treatments of the boundary conditions. Since, to our knowledge, these problems are currently unresolved, the analysis in the 
main text focuses on the trajectories within the physical model domain.

\section{REFERENCES}

Adlerman, E. J., K. K. Droegemeier, and R. P. Davies-Jones, 1999: A numerical simulation of cyclic mesocyclogenesis. J. Atmos. Sci., 56, 2045-2069, doi:10.1175/1520-0469(1999)056<2045: ANSOCM $>2.0 . C O ; 2$.

Aris, R., 1962: Vectors, Tensors, and the Basic Equations of Fluid Mechanics. Dover, 286 pp.

Batchelor, G. K., 2002: An Introduction to Fluid Dynamics. Cambridge University Press, 615 pp.

Brandes, E. A., 1981: Finestructure of the Del City-Edmond tornadic mesocirculation. Mon. Wea. Rev., 109, 635-647, doi:10.1175/1520-0493(1981)109<0635:FOTDCE > 2.0.CO;2.

Bryan, G. H., and J. M. Fritsch, 2002: A benchmark simulation for moist nonhydrostatic numerical models. Mon. Wea. Rev., 130, 2917-2928, doi:10.1175/1520-0493(2002)130<2917: ABSFMN $>2.0 . \mathrm{CO} ; 2$.

Bunkers, M. J., J. S. Johnson, L. J. Czepyha, J. M. Grywacz, B. A. Klimowski, and M. R. Hjelmfelt, 2006: An observational examination of long-lived supercells. Part II: Environmental conditions and forecasting. Wea. Forecasting, 21, 689-714, doi:10.1175/WAF952.1.

Casey, J., and P. M. Naghdi, 1991: On the Lagrangian description of vorticity. Arch. Ration. Mech. Anal., 115, 1-14, doi:10.1007/ BF01881677.

Dahl, J. M. L., M. D. Parker, and L. J. Wicker, 2012: Uncertainties in trajectory calculations within near-surface mesocyclones of simulated supercells. Mon. Wea. Rev., 140, 2959-2966, doi:10.1175/MWR-D-12-00131.1.

Davies-Jones, R. P., 1982: Observational and theoretical aspects of tornadogenesis. Intense Atmospheric Vortices, L. Bengtsson and J. Lighthill, Eds., Springer, 175-189.

, 1984: Streamwise vorticity: The origin of updraft rotation in supercell storms. J. Atmos. Sci., 41, 2991-3006, doi:10.1175/ 1520-0469(1984)041<2991:SVTOOU > 2.0.CO;2.

_ 2000: A Lagrangian model for baroclinic genesis of mesoscale vortices. Part I: Theory. J. Atmos. Sci., 57, 715-736, doi:10.1175/1520-0469(2000)057<0715:ALMFBG>2.0.CO;2.

, 2006: Integrals of the vorticity equation. Part I: General three- and two-dimensional flows. J. Atmos. Sci., 63, 598-610, doi:10.1175/JAS3646.1.

- 2008: Can a descending rain curtain in a supercell instigate tornadogenesis barotropically? J. Atmos. Sci., 65, 2469-2497, doi:10.1175/2007JAS2516.1.

— , and H. E. Brooks, 1993: Mesocyclogenesis from a theoretical perspective. The Tornado: Its Structure, Dynamics, Prediction, and Hazards, C. Church et al., Eds., Amer. Geophys. Union, 105-114.

— currents in sheared environments. J. Atmos. Sci., 70, 1204 1215, doi:10.1175/JAS-D-12-0149.1.

— , R. J. Trapp, and H. B. Bluestein, 2001: Tornadoes and tornadic storms. Severe Convective Storms, Meteor Monogr., No. 28, Amer. Meteor. Soc., 167-222.

Dawson, D. T., M. Xue, J. A. Milbrandt, and M. K. Yau, 2010: Comparison of evaporation and cold pool development between single-moment and multimoment bulk microphysics schemes in idealized simulations of tornadic thunderstorms. Mon. Wea. Rev., 138, 1152-1171, doi:10.1175/2009MWR2956.1.
Dutton, J. A., 1976: The Ceaseless Wind. McGraw-Hill, 579 pp.

Epifanio, C. C., and D. R. Durran, 2002: Lee-vortex formation in free-slip stratified flow over ridges. Part II: Mechanisms of vorticity and PV production in nonlinear viscous wakes. $J$. Atmos. Sci., 59, 1166-1181, doi:10.1175/1520-0469(2002)059<1166: LVFIFS $>2.0 . \mathrm{CO} ; 2$.

Gaudet, B. J., W. R. Cotton, and M. T. Montgomery, 2006: Lowlevel mesocyclonic concentration by nonaxisymmetric transport. Part II: Vorticity dynamics. J. Atmos. Sci., 63, 1134-1150, doi:10.1175/JAS3579.1.

Gilmore, M. S., J. M. Straka, and E. N. Rasmussen, 2004: Precipitation and evolution sensitivity in simulated deep convective storms: Comparisons between liquid-only and simple ice and liquid phase microphysics. Mon. Wea. Rev., 132, 1897-1916, doi:10.1175/1520-0493(2004)132<1897:PAESIS > 2.0.CO;2.

Kosiba, K., J. Wurman, Y. Richardson, P. Markowski, P. Robinson, and J. Marquis, 2013: Genesis of the Goshen County, Wyoming, tornado on 5 June 2009 during VORTEX2. Mon Wea. Rev., 141, 1157-1181, doi:10.1175/MWR-D-12-00056.1.

Kundu, P. K., and I. M. Cohen, 2008: Fluid Mechanics. Academic Press, 872 pp.

Markowski, P., and Y. Richardson, 2010: Mesoscale Meteorology in Midlatitudes. Wiley-Blackwell, $430 \mathrm{pp}$.

_ C. Hannon, J. Frame, E. Lancester, A. Pietrycha, R. Edwards, and R. L. Thompson, 2003a: Characteristics of vertical wind profiles near supercells obtained from the Rapid Update Cycle. Wea. Forecasting, 18, 1262-1272, doi:10.1175/ 1520-0434(2003)018<1262:COVWPN>2.0.CO;2.

_ J. M. Straka, and E. N. Rasmussen, 2003b: Tornadogenesis resulting from the transport of circulation by a downdraft: Idealized numerical simulations. J. Atmos. Sci., 60, 795-823, doi:10.1175/1520-0469(2003)060<0795:TRFTTO > 2.0.CO;2.

_ , Y. Richardson, E. Rasmussen, J. Straka, R. P. DaviesJones, and R. J. Trapp, 2008: Vortex lines within low-level mesocyclones obtained from pseudo-dual-Doppler radar observations. Mon. Wea. Rev., 136, 3513-3535, doi:10.1175/ 2008MWR2315.1.

— , and Coauthors, 2012: The pretornadic phase of the Goshen County, Wyoming, supercell of 5 June 2009 intercepted by VORTEX2. Part II: Intensification of low-level rotation. Mon Wea. Rev., 140, 2916-2938, doi:10.1175/MWR-D-11-00337.1.

Marquis, J., Y. Richardson, J. Wurman, and P. Markowski, 2008: Single- and dual-Doppler analysis of a tornadic vortex and surrounding storm-scale flow in the Crowell, Texas, supercell of 30 April 2000. Mon. Wea. Rev., 136, 5017-5043, doi:10.1175/ 2008MWR2442.1.

Mobbs, S. D., 1981: Some vorticity theorems and conservation laws for non-barotropic fluids. J. Fluid Mech., 108, 475-483, doi:10.1017/S002211208100222X.

Parker, M. D., 2012: Impacts of lapse rates on low-level rotation in idealized storms. J. Atmos. Sci., 69, 538-559, doi:10.1175/ JAS-D-11-058.1.

Rotunno, R., and J. B. Klemp, 1982: The influence of the shearinduced pressure gradient on thunderstorm motion. Mon. Wea. Rev., 110, 136-151, doi:10.1175/1520-0493(1982)110<0136: TIOTSI $>2.0 . \mathrm{CO} ; 2$

— supercell thunderstorms. J. Atmos. Sci., 42, 271-292, doi:10.1175/1520-0469(1985)042<0271:OTRAPO >2.0.CO;2.

Saffman, P. G., 1992: Vortex Dynamics. Cambridge Monographs on Mechanics and Applied Mathematics, Cambridge University Press, $311 \mathrm{pp}$. 
Salmon, R., 1998: Geophysical Fluid Dynamics. Oxford University Press, $378 \mathrm{pp}$.

Schenkman, A. D., M. Xue, and M. Hu, 2014: Tornadogenesis in a high-resolution simulation of the 8 May 2003 Oklahoma City supercell. J. Atmos. Sci., 71, 130-154, doi:10.1175/JAS-D-13-073.1.

Simmonds, J. G., 1994: A Brief on Tensor Analysis. Springer, 112 pp. Truesdell, C., 1954: The Kinematics of Vorticity. Indiana University Publications Science Series, No. 19, Indiana University Press, 232 pp.
Walko, R. L., 1993: Tornado spin-up beneath a convective cell: Required basic structure of the near-field boundary layer winds. The Tornado: Its Structure, Dynamics, Prediction, and Hazards, C. Church et al., Eds., Amer. Geophys. Union, 8995.

Wicker, L. J., and R. B. Wilhelmson, 1995: Simulation and analysis of tornado development and decay within a three-dimensional supercell thunderstorm. J. Atmos. Sci., 52, 2675-2703, doi:10.1175/1520-0469(1995)052<2675:SAAOTD>2.0.CO;2. 